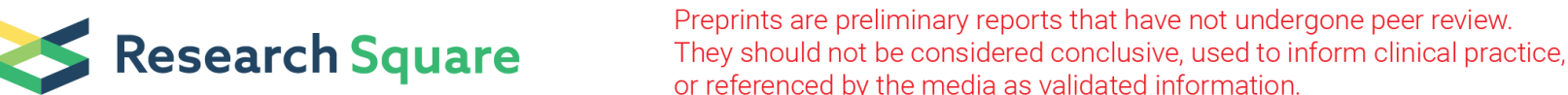 or referenced by the media as validated information.
}

\section{Multiple Accurate-Cubic Optical Solitons to the Kerr- Low and Power-low Nonlinear Schrodinger Equation without the Chromatic Dispersion}

\section{Emad H.M. Zahran}

Benha University

Ahmet Bekir ( $\sim$ bekirahmet@gmail.com )

Eskisehir https://orcid.org/0000-0001-9394-4681

\section{Research Article}

Keywords: the cubic-nonlinear Schrödinger equation, the extended simple equation method, the solitary wave ansatz method, the multiple accurate cubic optical solitons

Posted Date: May 17th, 2021

DOl: https://doi.org/10.21203/rs.3.rs-327900/v1

License: (c) This work is licensed under a Creative Commons Attribution 4.0 International License.

Read Full License 


\title{
Multiple Accurate-Cubic Optical Solitons to the Kerr-Low and Power-low Nonlinear Schrodinger Equation without the Chromatic Dispersion
}

\author{
Emad H.M. Zahran', Ahmet Bekir ${ }^{2, *}$ \\ ${ }^{1}$ Departmentof Mathematical and Physical Engineering,BenhaUniversity,Faculty of \\ Engineering, Shubra, Egypt \\ ${ }^{2}$ Neighbourhood of Akcaglan, Imarli Street, Number: 28/4, 26030, Eskisehir, Turkey \\ *Corresponding Author \\ E-mail: bekirahmet@gmail.com; emad.zahran@feng.bu.edu.eg
}

\begin{abstract}
The main target of this work is implementing multiple accurate cubic optical solitons for the nonlinear Schrödinger equation in the presence of third-order dispersion effects, absence of the chromatic dispersion. The emergence cubic optical solitons of the proposed model are extracted for the kerr-law and power law nonlinearity in the framework of two distinct techniques, the first one is the extended simple equation method (ESEM), while the other is the solitary wave ansatz method (SWAM). These cubic optical solitons for the kerr-law and power law nonlinearity have been extracted successfully at the same time and parallel via these two different techniques. A good comparison not only between our achieved results by these two manners but also with that achieved previously has been extracted .
\end{abstract}

Keywords: the cubic-nonlinear Schrödinger equation; the extended simple equation method; the solitary wave ansatz method; the multiple accurate cubic optical solitons

\section{1-Introduction}

Nonlinear optics explains nonlinear response of properties such as frequency, polarization, phase or path of incident light. These nonlinear interactions give rise to a host of optical phenomenas. Recently, many new paths have been suggested for enhanced nonlinearity and light manipulation, including twisted chromospheres, combining rich density of states with bond alternation, microscopic cascading of second-order nonlinearity, etc. Consequently, molecular nonlinear optics has been widely used in the biophotonics field, containing bioimaging, phototherapy, biosensing, etc.

In this study, we will implement the multiple accurate optical solitons for this kindly NLSE which plays principal rule in telecommunications engineering in the framework of the ESEM [1] and the SWAM [1-4]. The two proposed methods have been applied perfectly to achieve impressive accurate cubic optical solitons to this model with its kerr law and power law nonlinearity. The telecommunications engineering splits into wired communications that make use of underground communications cables and wireless communications that involve the transmission of information over a distance without help of wires. The arising problem for pure-quartic soliton related to the governing nonlinear Schrödinger equation can be nonintegrable one, for this reason we will propose the concept of cubic-quartic solitons in which the chromatic dispersion is replaced by the third order dispersion and fourth order dispersion together [5-11]. Specially, many studies in terms of various methods have been demonstrated 
through significant published articles that were extracted via many authors who discussed various forms of the cubic-quartic NLSE [11-20]. In the last few decades the dynamics of optical soliton cause surprise development in the telecommunications engineering [21-32]. Recently, few studies have been implemented to discuss the NLSE in the presence of the third order dispersion and absence of the chromatic dispersion [6-8], [33-34], while in the absence of third order dispersion and the chromatic dispersion this equation doesn't integrable [35].

According to [18], the suggested model can be proposed in the form,

$$
i Q_{t}+L\left|Q^{2}\right| Q+i a Q_{x x x}=i\left(v\left|Q^{2 m}\right| Q_{x}+\lambda\left(\left|Q^{2 m}\right| Q\right)_{x}+\mu\left(\left|Q^{2 m}\right|\right)_{x} Q\right)
$$

Where $Q(x, t)$ is the complex function that related to wave profile, $i=\sqrt{-1}$, and the other remaining constants appearing in this equation will be terminated to the following definitions $x, t$ are the spatial and temporal coordinates, $a$ denotes to the index of the third order dispersion, $\lambda$ is the index of self-gradient nonlinearity, $v, \mu$ related to profile of the higher-order dispersion effects, $m$ is the full nonlinearity variable and $F$ determines the nonlinear forms of refractive coefficient, where

$$
F\left(\left|Q^{2}\right|\right) Q \in \bigcup_{m, n=1}^{\infty} C^{k}\left((-n, n) \times(-m, m) ; R^{2}\right)
$$

The main target of this paper is to find multiple accurate cubic solitons for kerr-law and power-law of the NLSE in the framework of the ESEM and SWAM respectively,

\section{Mathematical analysis of the ESEM}

Any nonlinear evolution equation can be written in the form,

$$
R\left(h, h_{x}, h_{t}, h_{x x}, h_{t t}, \ldots \ldots\right)=0 .
$$

Where $\mathrm{R}$ is a function of $h(x, t)$ and its partial derivatives that involve the highest order derivatives and nonlinear terms, according to the transformation $h(x, t)=h(\zeta), \zeta=x-C_{0} t$ equation (2) can be reduced to the following ODE:

$$
S\left(h, h^{\prime}, h^{\prime \prime} \ldots \ldots \ldots . . . .\right)=0 \text {. }
$$

Where $S$ is a function in $h(\zeta)$ and its total derivatives, while ${ }^{\prime}=\frac{d}{d \zeta}$

The constructed solution according to this method is:

$$
\psi(\zeta)=\sum_{i=-m}^{m} A_{i} \varphi^{i}(\zeta)
$$

Where the positive integer $m$ in Eq. (4) can be located by balancing the highest order derivative term and the nonlinear term, while the arbitrary constants $A_{i}$ can be calculated later, the function $\phi(\zeta)$ satisfies the following new ansatz equation

$$
\varphi^{\prime}(\zeta)=a_{0}+a_{1} \varphi+a_{2} \varphi^{2} .
$$

While $a_{0}, a_{1}$ and $a_{2}$ admit the following two cases;

(1) If $a_{1}=a_{3}=0$, Eq.(5) will be transformed to the Riccati equation [30-31], which has the following solutions;

$$
\varphi(\zeta)=\frac{\sqrt{a_{0} a_{2}}}{a_{2}} \tan \left(\sqrt{a_{0} a_{2}}\left(\zeta+\zeta_{0}\right), a_{0} a_{2} \succ 0\right.
$$




$$
\varphi(\zeta)=\frac{\sqrt{-a_{0} a_{2}}}{a_{2}} \tanh \left(\sqrt{-a_{0} a_{2}} \zeta-\frac{\rho \ln \zeta_{0}}{2}\right), a_{0} a_{2} \prec 0, \zeta \succ 0, \rho= \pm 1
$$

(2) If $a_{0}=a_{3}=0$, Eq.(5) will be transformed to the Bernoulli equation [31], which has the following solutions;

$$
\begin{aligned}
& \varphi(\zeta)=\frac{a_{1} \operatorname{Exp}\left[a_{1}\left(\zeta+\zeta_{0}\right)\right]}{1-a_{2} \operatorname{Exp}\left[a_{1}\left(\zeta+\zeta_{0}\right)\right]}, a_{1} \succ 0 \\
& \varphi(\zeta)=\frac{-a_{1} \operatorname{Exp}\left[a_{1}\left(\zeta+\zeta_{0}\right)\right]}{1+a_{2} \operatorname{Exp}\left[a_{1}\left(\zeta+\zeta_{0}\right)\right]}, a_{1} \prec 0
\end{aligned}
$$

And the general solution to ansatz equation (5) is as follows:

$$
\begin{aligned}
& \varphi(\zeta)=-\frac{1}{a_{2}}\left(a_{1}-\sqrt{4 a_{1} a_{2}-a_{1}^{2}} \tan \left(\frac{\sqrt{4 a_{1} a_{2}-a_{1}^{2}}}{2}\left(\zeta+\zeta_{0}\right)\right), 4 a_{1} a_{2} \succ a_{1}^{2}, a_{2} \succ 0,\right. \\
& \varphi(\zeta)=\frac{1}{a_{2}}\left(a_{1}+\sqrt{4 a_{1} a_{2}-a_{1}^{2}} \tanh \left(\frac{\sqrt{4 a_{1} a_{2}-a_{1}^{2}}}{2}\left(\zeta+\zeta_{0}\right)\right), 4 a_{1} a_{2} \succ a_{1}^{2}, a_{2} \prec 0 .\right.
\end{aligned}
$$

Where $\zeta_{0}$ is the constancy of integration?

Finally, via inserting equation (4) into equation (5) and equating the coefficients of different powers of $\varphi^{i}$ to zero, we get a system of algebraic equations, by solving it we obtain the values of the unknown variable mentioned in these equations. Moreover, inserting these achieved variables into equation (5) then the aimed solutions will be extracted.

\section{The cubic optical solitons in the framework of the ESEM}

In this section, we will implement the ESEM to construct the cubic optical solitons for the kerr-law and power law nonlinearity of the suggested equation (1) mentioned above,

\subsection{The cubic optical solitons to the kerr-law nonlinearity,}

For the kerr-law nonlinearity Eq.(1) become,

$$
i Q_{t}+\left|Q^{2}\right| Q+i a Q_{x x x}=i\left(v\left|Q^{2}\right| Q_{x}+\lambda\left(\left|Q^{2}\right| Q\right)_{x}+\mu\left(\left|Q^{2}\right|\right)_{x} Q\right) .
$$

According to the suggested method, the solution is,

$$
Q(x, t)=\psi(\zeta) e^{i \eta(x, t)}, \zeta=k x+w t, \eta=q x+\delta t .
$$

Hence,

$$
\begin{aligned}
& Q_{t}=i \delta \psi e^{i \eta}+w \psi^{\prime} e^{i \eta}, \\
& Q_{x}=i q \psi e^{i \eta}+k \psi^{\prime} e^{i \eta}, \\
& Q_{x x}=-q^{2} \psi e^{i \eta}+2 i k q \psi^{\prime} e^{i \eta}+k^{2} \psi^{\prime \prime} e^{i \eta}, \\
& Q_{x x x}=-i q^{3} \psi e^{i \eta}-3 k q^{2} \psi^{\prime} e^{i \eta}+3 i q k^{2} \psi^{\prime \prime} e^{i \eta}+k^{3} \psi^{\prime \prime \prime} e^{i \eta},
\end{aligned}
$$




$$
\begin{aligned}
& \left|Q^{2}\right|=\psi^{2},\left(\left|Q^{2}\right|\right)_{x}=2 k \psi \psi^{\prime}, Q\left(\left|Q^{2}\right|\right)_{x}=2 k \psi^{2} \psi^{\prime} e^{i \eta}, \\
& \left(\left|Q^{2}\right| Q\right)_{x}=3 k \psi^{2} \psi^{\prime} e^{i \eta}+i q \psi^{3} e^{i \eta} .
\end{aligned}
$$

Via inserting the above relations into Eq. (12) we get the following real and imaginary parts respectively,

$$
\begin{aligned}
& \operatorname{Re}-3 a q k^{2} \psi^{\prime \prime}+(\lambda q+q v+1) \psi^{3}+\left(a q^{3}-\delta\right) \psi=0 . \\
& \operatorname{Im} a k^{3} \psi^{\prime \prime \prime}-k(3 \lambda+2 \mu-v) \psi^{2} \psi^{\prime}+\left(w-3 a k q^{2}\right) \psi^{\prime}=0 .
\end{aligned}
$$

Now, via integrating the imaginary part Eq. (21)with respect to $\zeta$, then it becomes approximately the same as Eq. (20) which represents the real part. For this reason we will implement the suggested method only for the real part which is,

$$
-3 a q k^{2} \psi^{\prime \prime}+(\lambda q+q v+1) \psi^{3}+\left(a q^{3}-\delta\right) \psi=0 .
$$

Via balancing $\psi^{\prime \prime}, \psi^{3}$ in Eq. (22) leads to $3 m=2 m+1$ which implies $m=1$, hence the solution is,

$$
\psi(\zeta)=\frac{A_{-1}}{\varphi}+A_{0}+A_{1} \varphi
$$

Where $\varphi^{\prime}=a_{0}+a_{1} \varphi+a_{2} \varphi^{2}+a_{3} \varphi^{3}$

Case 1: For the first family in which $a_{1}=a_{3}=0 \Rightarrow \varphi^{\prime}=a_{0}+a_{2} \varphi^{2}$ the solution is,

$$
\begin{aligned}
\psi^{\prime}= & -\frac{a_{0} A_{-1}}{\varphi^{2}}+A_{1} a_{0}+A_{1} a_{2} \varphi^{2}-a_{2} A_{-1} \\
\psi^{\prime \prime}= & \frac{2 a_{0}^{2} A_{-1}}{\varphi^{3}}+\frac{2 a_{0} a_{2} A_{-1}}{\varphi}+2 A_{1} a_{0} a_{2} \varphi+2 A_{1} a_{2}^{2} \varphi^{3} \\
\psi^{2}= & A_{1}^{2} \varphi^{2}+2 A_{0} A_{1} \varphi+\left(A_{0}^{2}+2 A_{-1} A_{1}\right)+\frac{A_{-1}^{2}}{\varphi^{2}}+\frac{2 A_{-1} A_{0}}{\varphi}, \\
\psi^{3}= & A_{1}^{3} \varphi^{3}+3 A_{0} A_{1}^{2} \varphi^{2}+\left(3 A_{1} A_{0}^{2}+3 A_{-1} A_{1}^{2}\right) \varphi+\left(A_{0}^{3}+6 A_{-1} A_{0} A_{1}\right) \\
& +\frac{A_{-1}^{3}}{\varphi^{3}}+\frac{3 A_{0} A_{-1}^{2}}{\varphi^{2}}+\frac{3 A_{-1} A_{0}^{2}+3 A_{1} A_{-1}^{2}}{\varphi},
\end{aligned}
$$

Via inserting the relations (23-27) into Eq. (22) and collecting and equating the coefficients of different powers of $\varphi^{i}$ to zero, lead to a system of algebraic equations and by solving this system the following results will be extracted,

$$
\begin{aligned}
& \text { (1) } a_{0}=\frac{-(\lambda q+q v+1) A_{-1}^{2}}{6 a q k^{2}}, a_{2}=\frac{-a q^{3}+\delta}{(\lambda q+q v+1) A_{-1}^{2}}, A_{0}=0, A_{1}=0, \\
& \text { (2) } a_{0}=\frac{-a q^{3}+\delta}{k A_{1} \sqrt{6 a q} \sqrt{\lambda q+q v+1}}, a_{2}=\frac{-A_{1} \sqrt{\lambda q+q v+1}}{k \sqrt{6 a q}}, A_{0}=0, A_{-1}=0, \\
& \text { (3) } a_{0}=\frac{a q^{3}-\delta}{k A_{1} \sqrt{6 a q} \sqrt{\lambda q+q v+1}}, a_{2}=\frac{A_{1} \sqrt{\lambda q+q v+1}}{k \sqrt{6 a q}}, A_{0}=0, A_{-1}=0,
\end{aligned}
$$




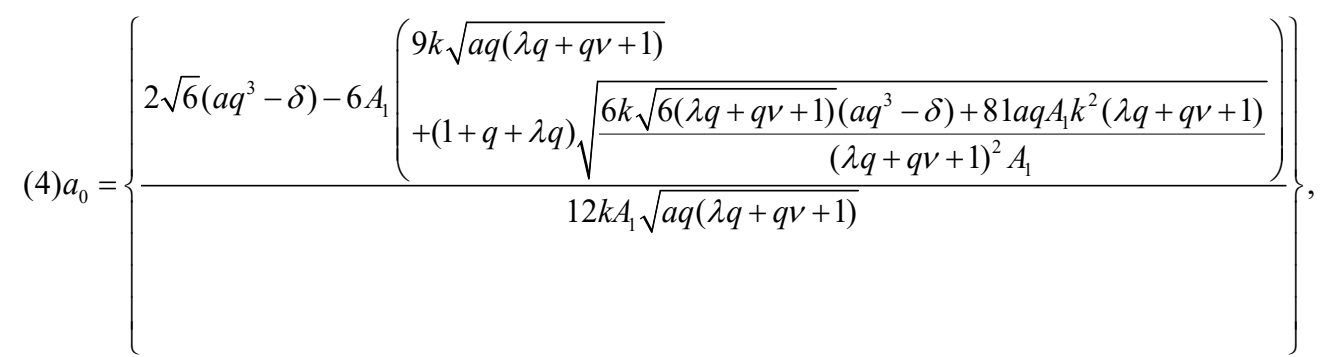

$a_{2}=\frac{A_{1} \sqrt{\lambda q+q v+1}}{k \sqrt{6 a q}}, A_{-1}=\frac{-k \sqrt{a q} \sqrt[3]{\frac{3}{2}}}{\sqrt{\lambda q+q v+1}}-\frac{1}{2} \sqrt{\frac{4 k \sqrt{6 a q}\left(a q^{3}-\delta\right)+54 a q k^{2} A_{1} \sqrt{\lambda q+q v+1}}{(\lambda q+q v+1)^{\frac{3}{2}} A_{1}}}, A_{0}=0$,

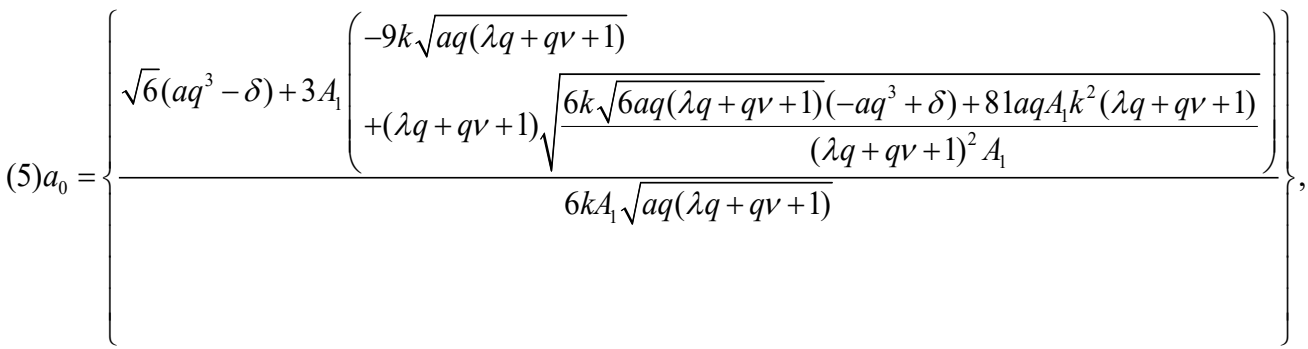

$a_{2}=\frac{A_{1} \sqrt{\lambda q+q v+1}}{k \sqrt{6 a q}}, A_{-1}=\frac{-3 k \sqrt{6 a q}+\sqrt{\lambda q+q v+1} \sqrt{\frac{4 k \sqrt{6 a q}\left(a q^{3}-\delta\right)+54 a q k^{2} A_{1} \sqrt{\lambda q+q v+1}}{(\lambda q+q v+1)^{\frac{3}{2}} A_{1}}}}{2 \sqrt{\lambda q+q v+1}}, A_{0}=0$,

$(6) a_{0}=\left\{\frac{\sqrt{6}\left(-a q^{3}+\delta\right)+3 A_{1}\left(\begin{array}{l}-9 k \sqrt{a q(\lambda q+q v+1)} \\ +(\lambda q+q v+1) \sqrt{\frac{6 k \sqrt{6(\lambda q+q v+1)}\left(a q^{3}-\delta\right)+81 a q A_{1} k^{2}(\lambda q+q v+1)}{(\lambda q+q v+1)^{2} A_{1}}}\end{array}\right)}{6 k A_{1} \sqrt{a q(\lambda q+q v+1)}}\right\}$

$a_{2}=\frac{-A_{1} \sqrt{\lambda q+q v+1}}{k \sqrt{6 a q}}, A_{-1}=\frac{3 k \sqrt{a q} \sqrt[3]{\frac{3}{2}}}{\sqrt{\lambda q+q v+1}}-\frac{1}{2} \sqrt{\frac{4 k \sqrt{6 a q}\left(a q^{3}-\delta\right)+54 a q k^{2} A_{1} \sqrt{\lambda q+q v+1}}{(\lambda q+q v+1)^{\frac{3}{2}} A_{1}}}, A_{0}=0$,

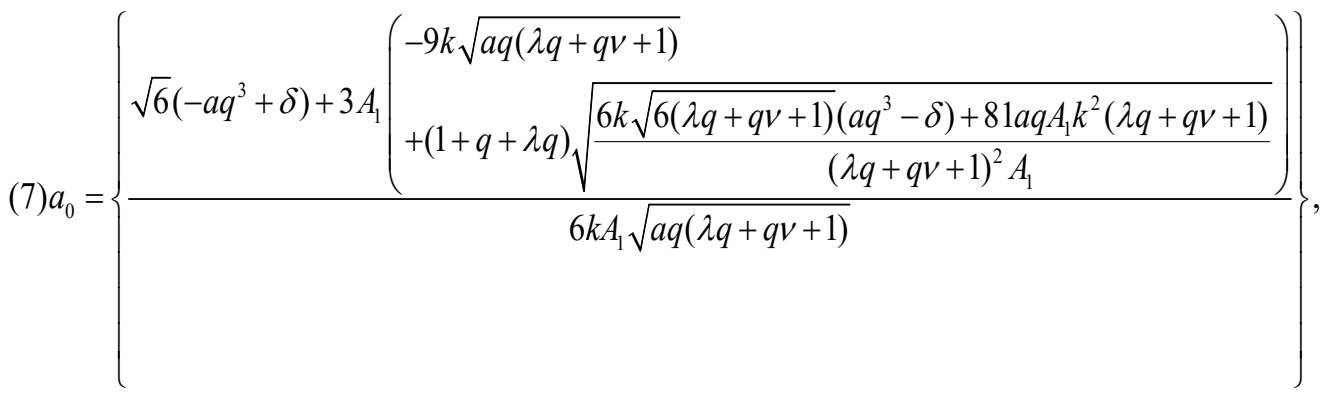

$a_{2}=\frac{-A_{1} \sqrt{\lambda q+q v+1}}{k \sqrt{6 a q}}, A_{-1}=\frac{3 k \sqrt{a q} \sqrt[3]{\frac{3}{2}}}{\sqrt{\lambda q+q v+1}}+\frac{1}{2} \sqrt{\frac{4 k \sqrt{6 a q}\left(a q^{3}-\delta\right)+54 a q k^{2} A_{1} \sqrt{\lambda q+q v+1}}{(\lambda q+q v+1)^{\frac{3}{2}} A_{1}}}, A_{0}=0$. 
These results imply 7-differents solutions, for simplicity we will extract the solutions corresponding to the first and the second result and plot them,

(3.1.1) for the 1-st result in which

$$
a_{0}=\frac{-(\lambda q+q v+1) A_{-1}^{2}}{6 a q k^{2}}, a_{2}=\frac{-a q^{3}+\delta}{(\lambda q+q v+1) A_{-1}^{2}}, A_{0}=0, A_{1}=0
$$

This solution can be simplified to be,

$$
A_{-1}=1, A_{0}=0, A_{1}=0, a_{0}=\frac{-5}{12}, a_{2}=\frac{-7}{5}, q=2, a=k=v=\lambda=\mu=\delta=1, \zeta_{0}=1
$$

According to the proposed method the solution is,

$$
\begin{aligned}
& \varphi(\zeta)=\frac{\sqrt{a_{0} a_{2}}}{a_{2}} \tan \left(\sqrt{a_{0} a_{2}}\left(\zeta+\zeta_{0}\right), a_{0} a_{2} \succ 0\right. \\
& \varphi(\zeta)=-1.1 \tan [0.8(x+t+1)] \\
& \psi(\zeta)=\frac{A_{-1}}{\varphi}+A_{0}+A_{1} \varphi \\
& \psi(\zeta)=-0.9 \cot [0.8(x+t+1)] \\
& Q(x, t)=\psi(\zeta) e^{i \eta(x, t)}, \zeta=k x+w t, \eta=q x+\delta t \\
& Q(x, t)=-0.9 \cot [0.8(x+t+1)] e^{i(2 x+t)} \\
& \operatorname{Re} Q(x, t)=-0.9 \cot [0.8(x+t+1)] \times \cos (2 x+t) \\
& \operatorname{Im} Q(x, t)=-0.9 \cot [0.8(x+t+1)] \times \sin (2 x+t)
\end{aligned}
$$

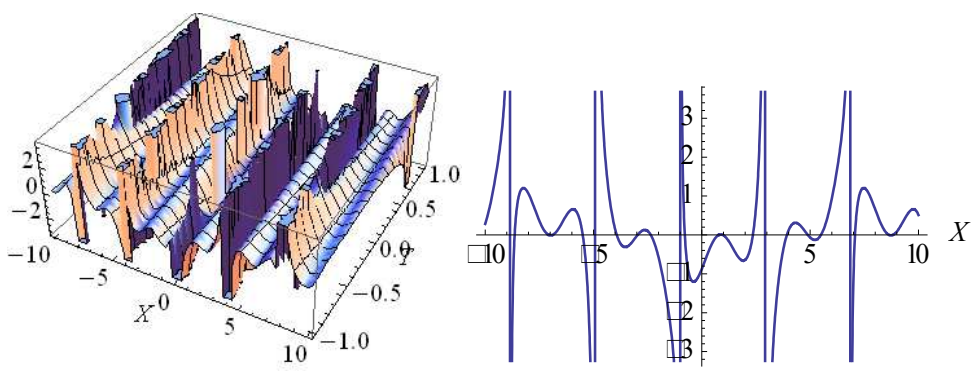

Figure 1. The cubic soliton of the real part Eq.(38) in $2 \mathrm{D}$ and $3 \mathrm{D}$ with values:

$A_{-1}=1, A_{0}=0, A_{1}=0, a_{0}=-0.42, a_{2}=-1.4, q=2, a=v=k=\lambda=\mu=\delta=1, \zeta_{0}=1$

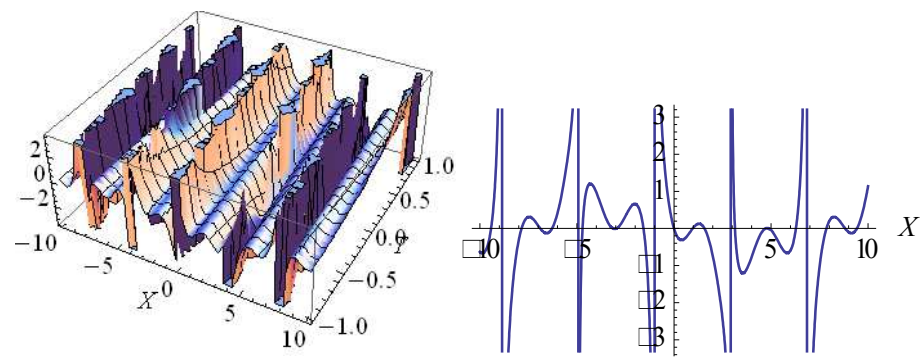

Figure 2. The cubic soliton the imaginary part Eq.(39) in 2D and 3D with values:

$$
A_{-1}=1, A_{0}=0, A_{1}=0, a_{0}=-0.42, a_{2}=-1.4, q=2, a=v=k=\lambda=\mu=\delta=1, \zeta_{0}=1
$$

(3.1.2) for the 2-nd result in which 
$a_{0}=\frac{-a q^{3}+\delta}{k A_{1} \sqrt{6 a q} \sqrt{\lambda q+q v+1}}, a_{2}=\frac{-A_{1} \sqrt{\lambda q+q v+1}}{k \sqrt{6 a q}}, A_{0}=0, A_{-1}=0$

This solution can be simplified to be,

$$
A_{-1}=0, A_{0}=0, A_{1}=1, a_{0}=-0.9, a_{2}=-0.7, q=2, a=v=k=\lambda=\mu=\delta=1, \zeta_{0}=1
$$

According to the proposed method the solution is,

$$
\begin{aligned}
& \varphi(\zeta)=\frac{\sqrt{a_{0} a_{2}}}{a_{2}} \tan \left(\sqrt{a_{0} a_{2}}\left(\zeta+\zeta_{0}\right), a_{0} a_{2} \succ 0\right. \\
& \varphi(\zeta)=-1.1 \tan [0.8(x+t+1)] \\
& \psi(\zeta)=\frac{A_{-1}}{\varphi}+A_{0}+A_{1} \varphi \\
& \psi(\zeta)=-1.1 \tan [0.8(x+t+1)] \\
& Q(x, t)=\psi(\zeta) e^{i \eta(x, t)}, \zeta=k x+w t, \eta=q x+\delta t \\
& Q(x, t)=-1.1 \tan [0.8(x+t+1)] e^{i(2 x+t)} \\
& \operatorname{Re} Q(x, t)=-1.1 \tan [0.8(x+t+1)] \times \cos (2 x+t) \\
& \operatorname{Im} Q(x, t)=-1.1 \tan [0.8(x+t+1)] \times \sin (2 x+t)
\end{aligned}
$$

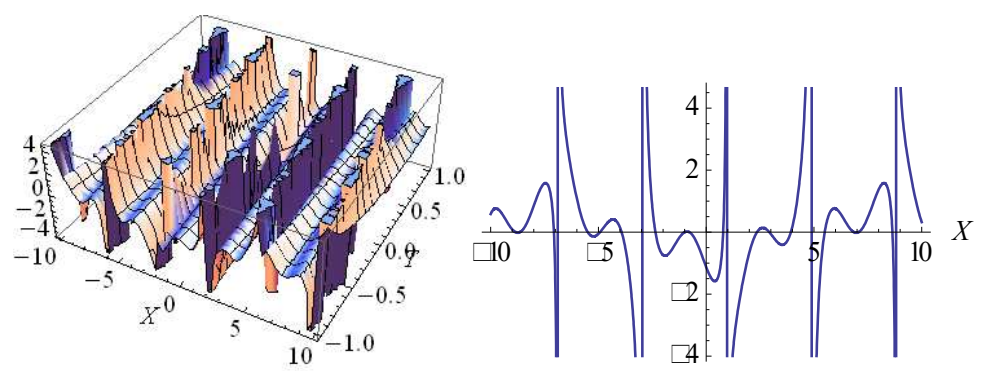

Figure 3. The cubic soliton of the real part Eq.(43) in 2D and 3D with values:

$$
A_{-1}=0, A_{0}=0, A_{1}=1, a_{0}=-0.9, a_{2}=-0.7, q=2, a=v=k=\lambda=\mu=\delta=1, \zeta_{0}=1
$$
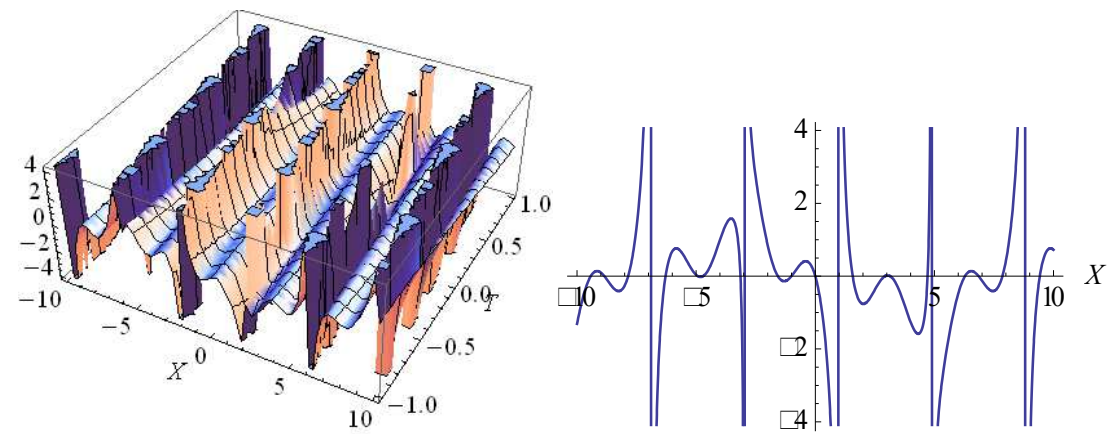

Figure 4. The cubic soliton the imaginary part Eq.(44) in 2D and 3D with values:

$A_{-1}=0, A_{0}=0, A_{1}=1, a_{0}=-0.9, a_{2}=-0.7, q=2, a=v=k=\lambda=\mu=\delta=1, \zeta_{0}=1$

In the same manner, we can plot the other cases.

Case 2: For the second family, in which $a_{0}=a_{3}=0 \Rightarrow \varphi^{\prime}=a_{1} \varphi+a_{2} \varphi^{2}$ the solution is, 


$$
\begin{aligned}
\psi^{\prime} & =A_{1} a_{2} \varphi^{2}+a_{1} A_{1} \varphi-\frac{A_{-1} a_{1}}{\varphi}-A_{-1} a_{2}, \\
\psi^{\prime \prime} & =2 A_{1} a_{2}^{2} \varphi^{3}+3 A_{1} a_{1} a_{2} \varphi^{2}+A_{1} a_{1}^{2} \varphi+A_{-1} a_{1} a_{2}+\frac{a_{1}^{2} A_{-1}}{\varphi}, \\
\psi^{2}= & A_{1}^{2} \varphi^{2}+2 A_{0} A_{1} \varphi+\left(A_{0}^{2}+2 A_{-1} A_{1}\right)+\frac{A_{-1}^{2}}{\varphi^{2}}+\frac{2 A_{-1} A_{0}}{\varphi}, \\
\psi^{3}= & A_{1}^{3} \varphi^{3}+3 A_{0} A_{1}^{2} \varphi^{2}+\left(3 A_{1} A_{0}^{2}+3 A_{-1} A_{1}^{2}\right) \varphi+\left(A_{0}^{3}+6 A_{-1} A_{0} A_{1}\right) \\
& +\frac{A_{-1}^{3}}{\varphi^{3}}+\frac{3 A_{0} A_{-1}^{2}}{\varphi^{2}}+\frac{3 A_{-1} A_{0}^{2}+3 A_{1} A_{-1}^{2}}{\varphi} .
\end{aligned}
$$

Via inserting the equations (45-48) into equation (22), hence collecting and equating the coefficients of different powers of $\varphi^{i}$ to zero, we obtain system of algebraic equations, by solving it we get these results,

$$
\begin{aligned}
& \text { (1) } q=\frac{-a q^{3}+\delta-(1+\lambda q) A_{0}^{2}}{A_{0}^{2}}, a_{1}=\frac{-\sqrt{\frac{2}{3}} \sqrt{-a q^{3}+\delta}}{k \sqrt{a q}}, a_{2}=\frac{-A_{1} \sqrt{-a q^{3}+\delta}}{k A_{0} \sqrt{a q}}, A_{-1}=0, \\
& \text { (2) } q=\frac{-a q^{3}+\delta-(1+\lambda q) A_{0}^{2}}{A_{0}^{2}}, a_{1}=\frac{\sqrt{\frac{2}{3}} \sqrt{-a q^{3}+\delta}}{k \sqrt{a q}}, a_{2}=\frac{A_{1} \sqrt{-a q^{3}+\delta}}{k A_{0} \sqrt{a q}}, A_{-1}=0, \\
& \text { (3) } q=-1-\lambda q, a_{1}=\frac{-i \sqrt{-a q^{3}+\delta}}{k \sqrt{3 a q}}, a_{2}=\frac{-i A_{0} \sqrt{-a q^{3}+\delta}}{k A_{-1} \sqrt{3 a q}}, A_{1}=0, \\
& \text { (4) } q=-1-\lambda q, a_{1}=\frac{i \sqrt{-a q^{3}+\delta}}{k \sqrt{3 a q}}, a_{2}=\frac{i A_{0} \sqrt{-a q^{3}+\delta}}{k A_{-1} \sqrt{3 a q}}, A_{1}=0,
\end{aligned}
$$

From which we can get 4-various solutions, we will extract the solutions corresponding to the first and third results and plot them.

(3.2.1) for the 1-st case in which

$$
q=\frac{-a q^{3}+\delta-(1+\lambda q) A_{0}^{2}}{A_{0}^{2}}, a_{1}=\frac{-\sqrt{\frac{2}{3}} \sqrt{-a q^{3}+\delta}}{k \sqrt{a q}}, a_{2}=\frac{-A_{1} \sqrt{-a q^{3}+\delta}}{k A_{0} \sqrt{a q}}, A_{-1}=0
$$

This result can be simplified to be,

$$
A_{-1}=0, A_{0}= \pm 1.2 i, A_{1}=1, a_{1}=-4 i, a_{2}= \pm 0.6 i, q=2, a=k=\lambda=\mu=\delta=1, \zeta_{0}=1 .
$$

Which adimts the following two different solutions the:

(3.2.1.1) when $A_{-1}=0, A_{0}=1.2 i, A_{1}=1, a_{1}=-4 i, a_{2}=0.6 i, q=2, a=k=\lambda=\mu=\delta=1, \zeta_{0}=1$.

$$
\begin{aligned}
& \varphi(\zeta)=\frac{\sqrt{a_{0} a_{2}}}{a_{2}} \tan \left(\sqrt{a_{0} a_{2}}\left(\zeta+\zeta_{0}\right), a_{0} a_{2} \succ 0\right. \\
& \varphi(\zeta)=-2.5 i \tan [1.8 x+1.8 t+1.8] \\
& \psi(\zeta)=\frac{A_{-1}}{\varphi}+A_{0}+A_{1} \varphi
\end{aligned}
$$




$$
\begin{aligned}
& \psi(\zeta)=i(1.2-2.5 \tan [1.8 x+1.8 t+1.8]) \\
& Q(x, t)=\psi(\zeta) e^{i \eta(x, t)}, \zeta=k x+w t, \eta=q x+\delta t \\
& Q(x, t)=i(1.2-2.5 \tan [1.8 x+1.8 t+1.8]) e^{i(2 x+t)} \\
& \operatorname{Re} Q(x, t)=-(1.2-2.5 \tan [1.8 x+1.8 t+1.8]) \times \sin (2 x+t) \\
& \operatorname{Im} Q(x, t)=(1.2-2.5 \tan [1.8 x+1.8 t+1.8]) \times \cos (2 x+t)
\end{aligned}
$$
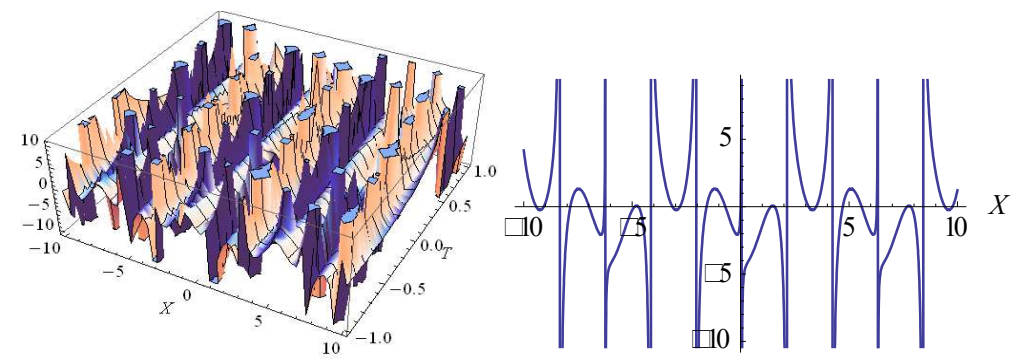

Figure 5. The cubic soliton for the real part Eq.(53) in 2D and 3D with values: $A_{-1}=0, A_{0}=1.2 i, A_{1}=1, a_{1}=-4 i, a_{2}=0.6 i, q=2, a=k=\lambda=\mu=\delta=1, \zeta_{0}=1$.
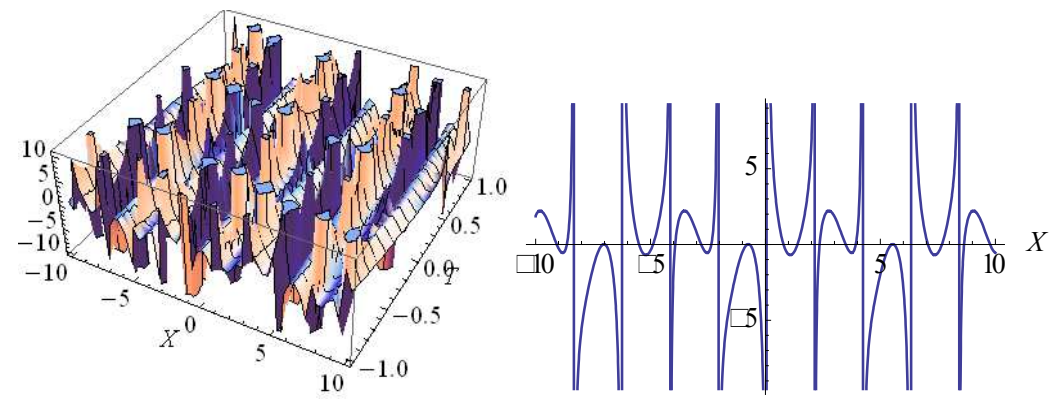

Figure 6. The cubic soliton for the imaginary part Eq.(54) in $2 \mathrm{D}$ and $3 \mathrm{D}$ with values: $A_{-1}=0, A_{0}=1.2 i, A_{1}=1, a_{1}=-4 i, a_{2}=0.6 i, q=2, a=k=\lambda=\mu=\delta=1, \zeta_{0}=1$.

(3.2.1.2) when $A_{-1}=0, A_{0}=1.2 i, A_{1}=1, a_{1}=-4 i, a_{2}=-0.6 i, q=2, a=k=\lambda=\mu=\delta=1, \zeta_{0}=1$.

$$
\begin{aligned}
& \varphi(\zeta)=\frac{-a_{1} \operatorname{Exp}\left[a_{1}\left(\zeta+\zeta_{0}\right)\right]}{1+a_{2} \operatorname{Exp}\left[a_{1}\left(\zeta+\zeta_{0}\right)\right]}, a_{1} \prec 0 \\
& \varphi(\zeta)=\frac{0.4 i \operatorname{Exp}[-0.4 i(x+t+1)]}{1-0.6 i \operatorname{Exp}[-0.4 i(x+t+1)]} \\
& \psi(\zeta)=\frac{A_{-1}}{\varphi}+A_{0}+A_{1} \varphi \\
& \psi(\zeta)=1.2 i+\frac{0.4 i \operatorname{Exp}[-0.4 i(x+t+1)]}{1-0.6 i \operatorname{Exp}[-0.4 i(x+t+1)]} \\
& Q(x, t)=\psi(\zeta) e^{i \eta(x, t)}, \zeta=k x+w t, \eta=q x+\delta t \\
& Q(x, t)=\left(1.2 i+\frac{0.4 i \operatorname{Exp}[-0.4 i(x+t+1)]}{1-0.6 i \operatorname{Exp}[-0.4 i(x+t+1)]}\right) e^{i(2 x+t)}
\end{aligned}
$$




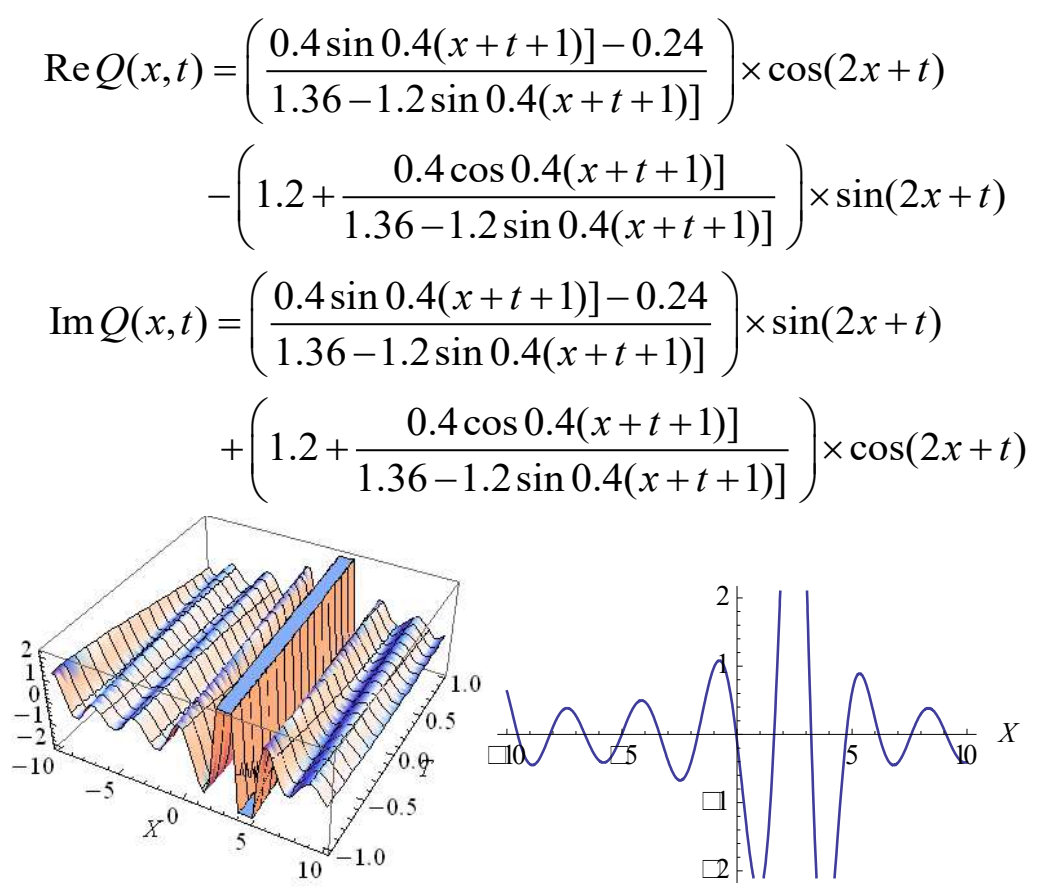

Figure 7. The cubic soliton for the real part Eq.(58) in $2 \mathrm{D}$ and $3 \mathrm{D}$ with values: $A_{-1}=0, A_{0}=1.2 i, A_{1}=1, a_{1}=-4 i, a_{2}=-0.6 i, q=2, a=k=\lambda=\mu=\delta=1, \zeta_{0}=1$.
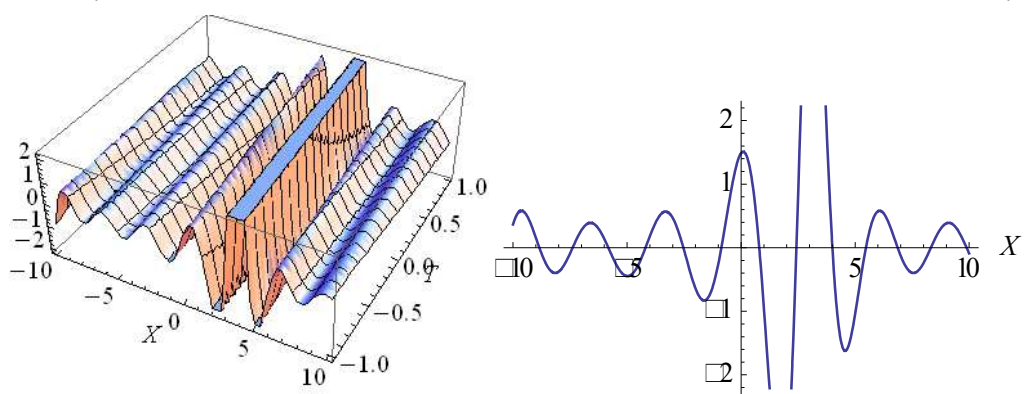

Figure 8. The cubic soliton for the imaginary part Eq.(59) in $2 \mathrm{D}$ and $3 \mathrm{D}$ with values: $A_{-1}=0, A_{0}=1.2 i, A_{1}=1, a_{1}=-4 i, a_{2}=-0.6 i, q=2, a=k=\lambda=\mu=\delta=1, \zeta_{0}=1$.

(3.2.2) for the 3-th case in which

$$
q=-1-\lambda q, a_{1}=\frac{-i \sqrt{-a q^{3}+\delta}}{k \sqrt{3 a q}}, a_{2}=\frac{-i A_{0} \sqrt{-a q^{3}+\delta}}{k A_{-1} \sqrt{3 a q}}, A_{1}=0,
$$

This result can be simplified to be,

$$
\begin{aligned}
& A_{-1}=1, A_{0}=1, A_{1}=0, a_{1}=1.1, a_{2}=1.1, q=2, a=k=\mu=\delta=\zeta_{0}=1, \lambda=-1.5 . \\
& \varphi(\zeta)=\frac{\sqrt{a_{0} a_{2}}}{a_{2}} \tan \left(\sqrt{a_{0} a_{2}}\left(\zeta+\zeta_{0}\right), a_{0} a_{2} \succ 0\right. \\
& \varphi(\zeta)=0.9 \tan [1.02 x+1.02 t+1.02] \\
& \psi(\zeta)=\frac{A_{-1}}{\varphi}+A_{0}+A_{1} \varphi \\
& \psi(\zeta)=1+1.1 \cot [1.02 x+1.02 t+1.02]
\end{aligned}
$$




$$
\begin{aligned}
& Q(x, t)=\psi(\zeta) e^{i \eta(x, t)}, \zeta=k x+w t, \eta=q x+\delta t \\
& Q(x, t)=(1+1.1 \cot [1.02 x+1.02 t+1.02]) \times e^{i(2 x+t)} \\
& \operatorname{Re} Q(x, t)=(1+1.1 \cot [1.02 x+1.02 t+1.02]) \times \cos (2 x+t) \\
& \operatorname{Im} Q(x, t)=(1+1.1 \cot [1.02 x+1.02 t+1.02]) \times \sin (2 x+t)
\end{aligned}
$$
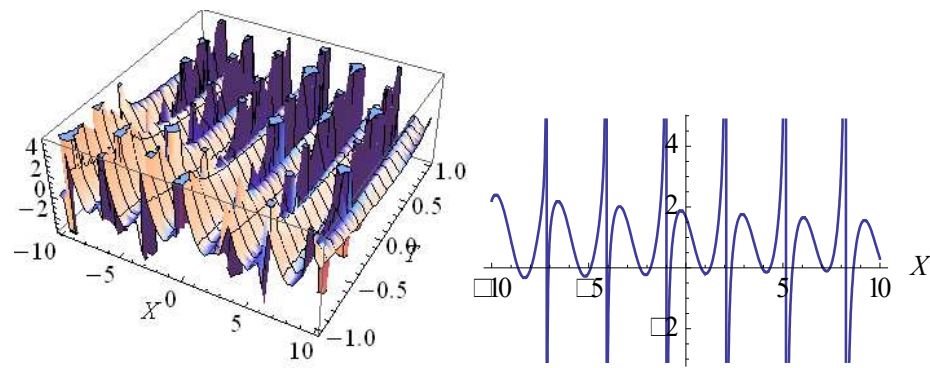

Figure 9. The cubic soliton for the real part Eq.(63) in 2D and 3D with values: $A_{-1}=0, A_{0}=1.2 i, A_{1}=1, a_{1}=-4 i, a_{2}=0.6 i, q=2, a=k=\lambda=\mu=\delta=1, \zeta_{0}=1$.
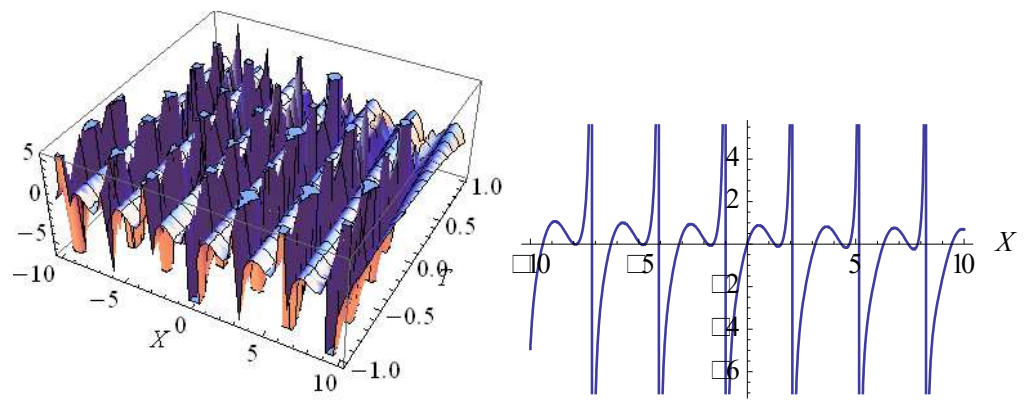

Figure 10. The cubic soliton for the imaginary part Eq.(64) in 2D and 3D with values:

$$
A_{-1}=0, A_{0}=1.2 i, A_{1}=1, a_{1}=-4 i, a_{2}=0.6 i, q=2, a=k=\lambda=\mu=\delta=1, \zeta_{0}=1 \text {. }
$$

In the same manner, we can plot the other cases.

By the same manner we can implement the first and second family of the suggested method for the imaginary part of the kerr-low nonlinearity Eq. (21) to extract the cubic- solitons.

\subsection{The cubic optical soliton to the power-law nonlinearity,}

For the power-law nonlinearity Eq.(1) is,

$$
i Q_{t}+\left|Q^{2 m}\right| Q+i a Q_{x x x}=i\left(v\left|Q^{2 m}\right| Q_{x}+\lambda\left(\left|Q^{2 m}\right| Q\right)_{x}+\mu\left(\left|Q^{2 m}\right|\right)_{x} Q\right)
$$

Via inserting the relations (13-19) into equation (65), then it will be transformed to the following real and imaginary equations respectively:

$$
\begin{aligned}
& \operatorname{Re}-3 a q k^{2} \psi^{\prime \prime}+\left(a q^{3}-\delta\right) \psi+(\lambda q+q v+1) \psi^{2 m+1}=0 . \\
& \operatorname{Im} a k^{3} \psi^{\prime \prime \prime}+\left(w-3 a k q^{2}\right) \psi^{\prime}-\frac{k[v-\lambda(2 m+1)]}{2 m+1} \psi^{2 m+1}--\frac{\mu(2 m+1)}{2 m+2} \psi^{2 m+2}=0 .
\end{aligned}
$$

Firstly, we will implement the suggested method for the real part Eq. (66),

$$
-3 a q k^{2} \psi^{\prime \prime}+\left(a q^{3}-\delta\right) \psi+(\lambda q+q v+1) \psi^{2 m+1}=0
$$


Via applying the homogeneous balance between $\varphi^{\prime \prime}$ and $\varphi^{2 m+1}$ leads to $N+2=(2 m+1) N$ which implies that $N=\frac{1}{2 m}$, consequently let us take the new transformation $\psi=V^{\frac{1}{2 m}}$ via which this equation will be transformed to,

$$
\frac{-3 a q k^{2}}{2 m} V V^{\prime \prime}+\left(a q^{3}-\delta\right) V^{2}+(\lambda q+q v+1) V^{3}-\frac{3 a q k^{2}(1-2 m)}{4 m^{2}}=0
$$

Now, by applying the homogeneous balance between $V V^{\prime \prime}$ and $V^{3}$ it will lead to $N=2$, hence the solution in the framework of the proposed method must be in the form,

$$
V(\zeta)=\frac{A_{-2}}{\varphi^{2}}+\frac{A_{-1}}{\varphi}+A_{0}+A_{1} \varphi+A_{2} \varphi^{2}
$$

Case 1: The first family, in which $a_{1}=a_{3}=0 \Rightarrow \varphi^{\prime}=a_{0}+a_{2} \varphi^{2}$ the solution is,

$$
\begin{aligned}
V^{\prime}= & \frac{-2 a_{0} A_{-2}}{\varphi^{3}}-\frac{a_{0} A_{-1}}{\varphi^{2}}-\frac{2 a_{2} A_{-2}}{\varphi}+\left(A_{1} a_{0}-a_{2} A_{-1}\right)+2 A_{2} a_{0} \varphi+\left(A_{1} a_{2}+2 a_{2} A_{2}\right) \varphi^{2} . \\
V^{\prime \prime}= & \frac{6 a_{0}^{2} A_{-2}}{\varphi^{4}}+\frac{2 a_{0}^{2} A_{-1}}{\varphi^{3}}+\frac{8 a_{0} a_{2} A_{-2}}{\varphi^{2}}+\frac{2 a_{0} a_{2} A_{1}}{\varphi}+2\left(A_{2} a_{0}^{2}+A_{-2} a_{2}^{2}\right) \\
& +2 a_{0} a_{2} A_{2} \varphi^{2}+2 a_{0} a_{2}\left(A_{1}+A_{2}\right) \varphi+2 a_{2}^{2}\left(A_{1}+A_{2}\right) \varphi^{3} .
\end{aligned}
$$

By inserting $V V^{\prime \prime}, V^{3}$ and $V^{2}$ into Eq. (66), by substituting the same values of parameters used above and solving the extracted system we obtain a set of results from which only two are suitable and the others will be refused because either $a_{0}=0$ or $a_{2}=0$ or both.

The two suitable results are,

$$
\begin{aligned}
& \text { (1) } A_{-2}=\frac{36 a_{0}^{2}}{5}, a_{2}=\frac{-7}{24 a_{0}^{2}}, A_{0}=-2.1, A_{-1}=A_{1}=A_{2}=0, m=0.5 \\
& \text { (2) } A_{-2}=\frac{36 a_{0}^{2}}{5}, a_{2}=\frac{7}{24 a_{0}^{2}}, A_{0}=0.7, A_{-1}=A_{1}=A_{2}=0, m=0.5
\end{aligned}
$$

Now we will plot only one of these two similar results, say the first result which is:

$$
A_{-2}=\frac{36 a_{0}^{2}}{5}, a_{2}=\frac{-7}{24 a_{0}^{2}}, A_{0}=-2.1, A_{-1}=A_{1}=A_{2}=0, m=0.5
$$

This solution can be simplified to be:

$$
a_{0}= \pm 0.4, a_{2}= \pm 0.1, A_{0}=-2.1, A_{-2}=1, A_{-1}=A_{1}=A_{2}=0, m=0.5
$$

From which we get four solutions, for similarity we will choose only two of them

(1)when $a_{0}=0.4, a_{2}=0.1, A_{0}=-2.1, A_{-2}=1, A_{-1}=A_{1}=A_{2}=0, m=0.5$

In the framework of the suggested method the solution is,

$$
\begin{aligned}
& \varphi(\zeta)=\frac{\sqrt{a_{0} a_{2}}}{a_{2}} \tan \left(\sqrt{a_{0} a_{2}}\left(\zeta+\zeta_{0}\right), a_{0} a_{2} \succ 0\right. \\
& \varphi(\zeta)=2 \tan [0.2 x+0.2 t+0.2] \\
& V(\zeta)=\frac{A_{-2}}{\varphi^{2}}+\frac{A_{-1}}{\varphi}+A_{0}+A_{1} \varphi+A_{2} \varphi^{2}
\end{aligned}
$$




$$
\begin{aligned}
& V(\zeta)=\frac{1}{4}(\cot [0.2 x+0.2 t+0.2])^{2}-2.1 \\
& \psi(\zeta)=\left(\frac{1}{4}(\cot [0.2 x+0.2 t+0.2])^{2}-2.1\right)^{\frac{1}{2 m}} \\
& Q(x, t)=\left(\frac{1}{4}(\cot [0.2 x+0.2 t+0.2])^{2}-2.1\right)^{\frac{1}{2 m}} e^{i(2 x+t)} \\
& \operatorname{Re} Q(x, t)=\left(\frac{1}{4}(\cot [0.2 x+0.2 t+0.2])^{2}-2.1\right)^{\frac{1}{2 m}} \cos (2 x+t) \\
& \operatorname{Im} Q(x, t)=\left(\frac{1}{4}(\cot [0.2 x+0.2 t+0.2])^{2}-2.1\right)^{\frac{1}{2 m}} \sin (2 x+t)
\end{aligned}
$$
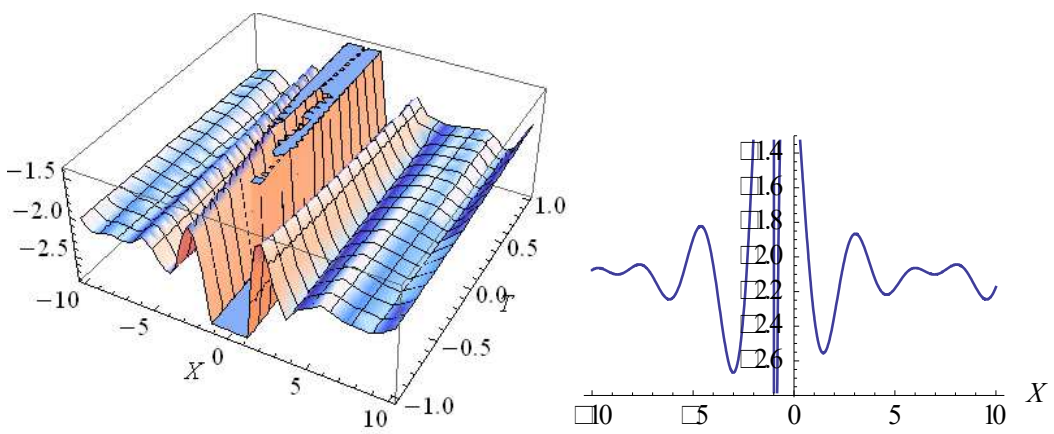

Figure 11. The cubic-soliton of the real part Eq.(79) in 2D and 3D with values: $\zeta_{0}=1$

$$
a_{0}=0.4, a_{2}=0.1, A_{0}=-2.1, A_{-2}=1, A_{-1}=A_{1}=A_{2}=0, m=0.5
$$
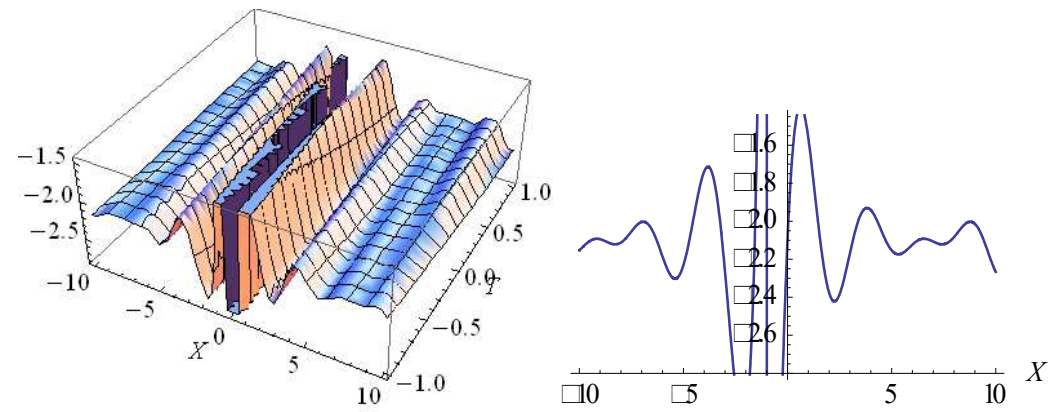

Figure 12. The cubic-soliton of the imaginary part Eq.(80) in $2 \mathrm{D}$ and $3 \mathrm{D}$ with values: $\zeta_{0}=1$

$$
a_{0}=0.4, a_{2}=0.1, A_{0}=-2.1, A_{-2}=1, A_{-1}=A_{1}=A_{2}=0, m=0.5
$$

(2) when $a_{0}=-0.4, a_{2}=0.1, A_{0}=-2.1, A_{-2}=1, A_{-1}=A_{1}=A_{2}=0, m=0.5$

In the framework of the suggested method the solution is,

$$
\begin{aligned}
& \varphi(\zeta)=\frac{\sqrt{-a_{0} a_{2}}}{a_{2}} \tanh \left(\sqrt{-a_{0} a_{2}} \zeta-\frac{\rho \ln \zeta_{0}}{2}\right), a_{0} a_{2} \prec 0, \zeta \succ 0, \rho= \pm 1 \\
& \varphi(\zeta)=2 \tanh [0.2 x+0.2 t+0.3] .
\end{aligned}
$$




$$
\begin{aligned}
& V(\zeta)=\frac{A_{-2}}{\varphi^{2}}+\frac{A_{-1}}{\varphi}+A_{0}+A_{1} \varphi+A_{2} \varphi^{2} \\
& V(\zeta)=\frac{1}{4}(\operatorname{coth}[0.2 x+0.2 t+0.3])^{2}-2.1 \\
& \psi(\zeta)=\left(\frac{1}{4}(\operatorname{coth}[0.2 x+0.2 t+0.3])^{2}-2.1\right)^{\frac{1}{2 m}} \\
& Q(x, t)=\left(\frac{1}{4}(\operatorname{coth}[0.2 x+0.2 t+0.3])^{2}-2.1\right)^{\frac{1}{2 m}} e^{i(2 x+t)} \\
& \operatorname{Re} Q(x, t)=\left(\frac{1}{4}(\operatorname{coth}[0.2 x+0.2 t+0.3])^{2}-2.1\right)^{\frac{1}{2 m}} \cos (2 x+t) \\
& \operatorname{Im} Q(x, t)=\left(\frac{1}{4}(\operatorname{coth}[0.2 x+0.2 t+0.2])^{2}-2.1\right)^{\frac{1}{2 m}} \sin (2 x+t)
\end{aligned}
$$
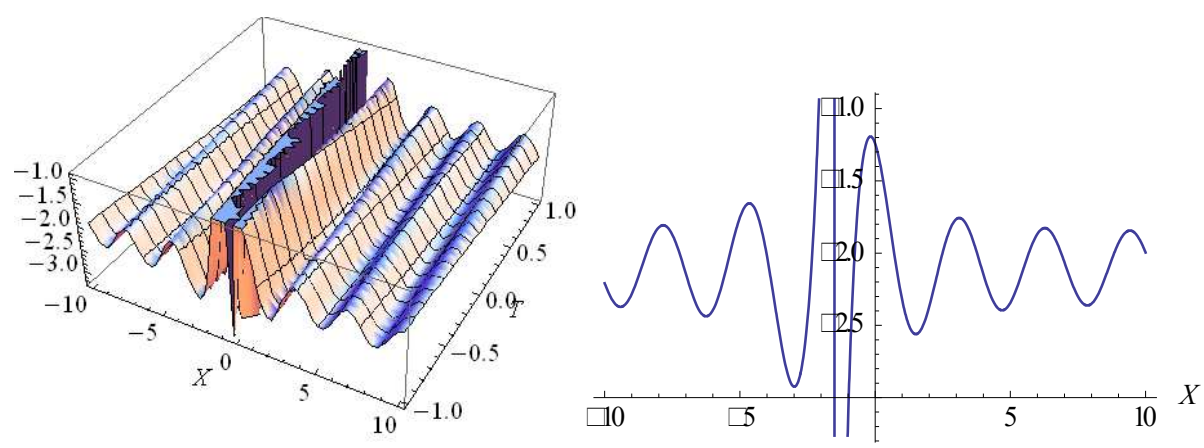

Figure 13. The cubic-soliton of the real part Eq.(85) in 2D and 3D with values: $\zeta_{0}=2, \rho=-1$

$$
a_{0}=-0.4, a_{2}=0.1, A_{0}=-2.1, A_{-2}=1, A_{-1}=A_{1}=A_{2}=0, m=0.5
$$
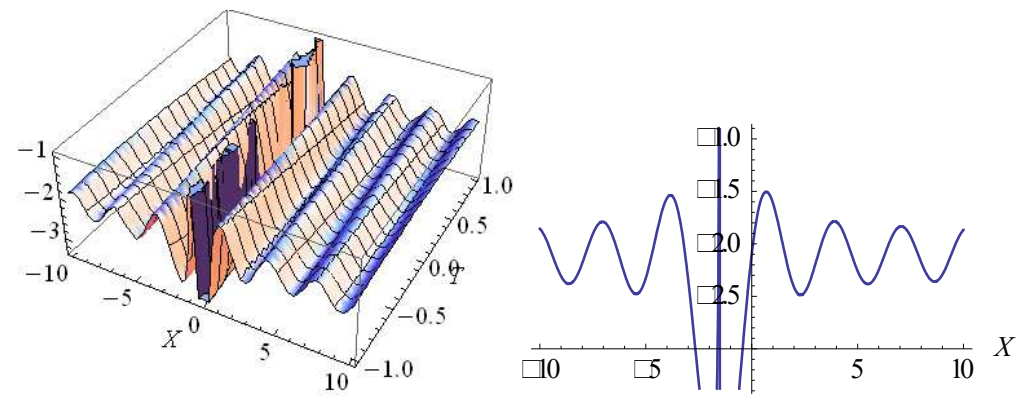

Figure 14. The cubic-soliton of the imaginary part Eq.(86) in $2 \mathrm{D}$ and $3 \mathrm{D}$ with values: $\zeta_{0}=2, \rho=-1$

$$
a_{0}=-0.4, a_{2}=0.1, A_{0}=-2.1, A_{-2}=1, A_{-1}=A_{1}=A_{2}=0, m=0.5
$$

Case 2: The second family, in which $a_{0}=a_{3}=0 \Rightarrow \varphi^{\prime}=a_{1} \varphi+a_{2} \varphi^{2}$ the solution is,

$$
\begin{aligned}
V^{\prime}= & \frac{-2 a_{1} A_{-2}}{\varphi^{2}}-\frac{2 a_{2} A_{-2}+a_{1} A_{-1}}{\varphi}-a_{2} A_{-1}+A_{1} a_{1} \varphi+\left(A_{1} a_{2}+2 a_{1} A_{2}\right) \varphi^{2}+2 a_{2} A_{2} \varphi^{3} \\
V^{\prime \prime}= & \frac{4 a_{1} A_{-2}}{\varphi^{3}}+\frac{2 a_{2} A_{-2}+a_{1} A_{-1}}{\varphi^{2}}+A_{1} a_{1}^{2} \varphi+\left(3 a_{1} a_{2} A_{1}+4 A_{2} a_{1}^{2}\right) \varphi^{2} \\
& +\left(2 a_{2}^{2} A_{1}+6 a_{1} a_{2} A_{2}\right) \varphi^{3}+3 a_{2}^{2} A_{2} \varphi^{4} .
\end{aligned}
$$


Via inserting $V V^{\prime \prime}, V^{3}$ and $V^{2}$ into Eq. (66) and using the same values of parameters used above we get system of equations in which the first equation implies that $A_{-2}=0$, by inserting about it in the other remaining equations and solving them we get big number of similar complicated results, for similarity we will study only one of these results which is,

$$
\begin{aligned}
& a_{1}=2, a_{2}=\frac{1}{3}(9+\sqrt{21}), A_{-1}=\frac{7\left\{\sqrt{6}(23+7 \sqrt{21})^{1.5}-46 \sqrt{6(23+7 \sqrt{21})}\right\}}{10000}, A_{0}=-0.7, \\
& A_{1}=\frac{7 \sqrt{\frac{1}{6}(23+7 \sqrt{21})}}{10}, A_{2}=\frac{\left\{\sqrt{6}(23+7 \sqrt{21})^{1.5}-30 \sqrt{6(23+7 \sqrt{21})}\right\}}{360}, m=\frac{4}{7} \sqrt{6(23+7 \sqrt{21})} .
\end{aligned}
$$

This solution can be simplified to be,

$$
A_{-2}=0, A_{-1}=0.1, A_{0}=-0.7, a_{1}=2, a_{2}=4.5, A_{1}=2.1, A_{2}=1.3, m=10.4
$$

In the framework of the suggested method the solution is,

$$
\begin{gathered}
\varphi(\zeta)=\frac{a_{1} \operatorname{Exp}\left[a_{1}\left(\zeta+\zeta_{0}\right)\right]}{1-a_{2} \operatorname{Exp}\left[a_{1}\left(\zeta+\zeta_{0}\right)\right]}, a_{1} \succ 0 \\
\varphi(\zeta)=\frac{2 \operatorname{Exp}[2 x+2 t+2]}{1-4.5 \operatorname{Exp}[2 x+2 t+2]} \\
V(\zeta)=\frac{A_{-2}}{\varphi^{2}}+\frac{A_{-1}}{\varphi}+A_{0}+A_{1} \varphi+A_{2} \varphi^{2} \\
V(\zeta)=\frac{0.1(1-4.5 \operatorname{Exp}[2 x+2 t+2])}{2 \operatorname{Exp}[2 x+2 t+2]}+\frac{3.4 \operatorname{Exp}[2 x+2 t+2]}{1-4.5 \operatorname{Exp}[2 x+2 t+2]}-0.7 \\
\psi(\zeta)=\left(\frac{0.1(1-4.5 \operatorname{Exp}[2 x+2 t+2])}{2 \operatorname{Exp}[2 x+2 t+2]}+\frac{3.4 \operatorname{Exp}[2 x+2 t+2]}{1-4.5 \operatorname{Exp}[2 x+2 t+2]}-0.7\right)^{\frac{1}{2 m}} \\
\operatorname{Im} Q(\zeta)=\left(\frac{(1-4.5 \operatorname{Exp}[2 x+2 t+2])}{20 \operatorname{Exp}[2 x+2 t+2]}+\frac{3.4 \operatorname{Exp}[2 x+2 t+2]}{1-4.5 \operatorname{Exp}[2 x+2 t+2]}-0.7\right)^{\frac{1}{2 m}} \sin (2 x+t) \\
\operatorname{Re} Q(\zeta)=\left(\frac{(1-4.5 \operatorname{Exp}[2 x+2 t+2])}{20 \operatorname{Exp}[2 x+2 t+2]}+\frac{3.4 \operatorname{Exp}[2 x+2 t+2]}{1-4.5 \operatorname{Exp}[2 x+2 t+2]}-0.7\right)^{\frac{1}{2 m}} \cos (2 x+t) \\
2 \operatorname{Exp}[2 x+2 t+2]
\end{gathered}
$$



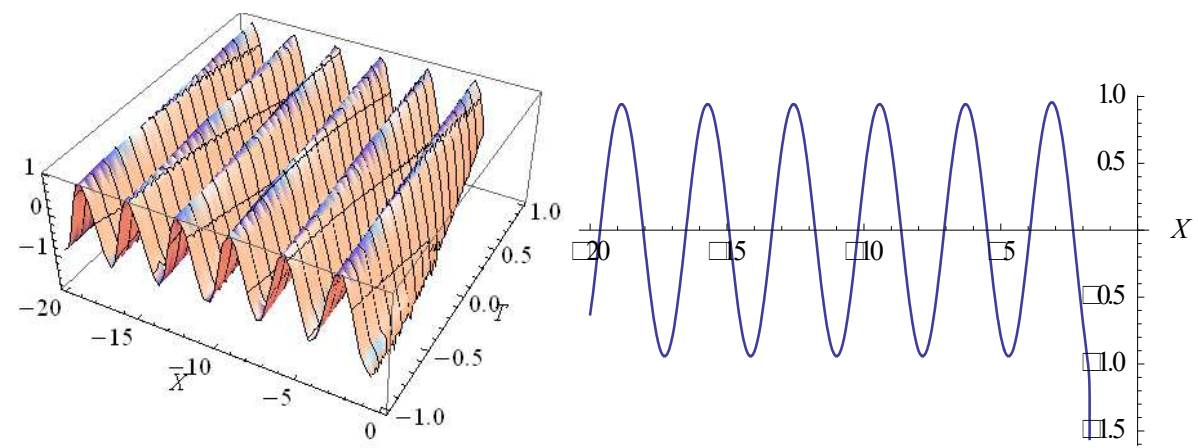

Figure 15. The cubic-soliton of the real part Eq.(95) in 2D and 3D with values: $\zeta_{0}=1$ $A_{-2}=0, A_{-1}=0.1, A_{0}=-0.7, a_{1}=2, a_{2}=4.5, A_{1}=2.1, A_{2}=1.3, m=10.4$
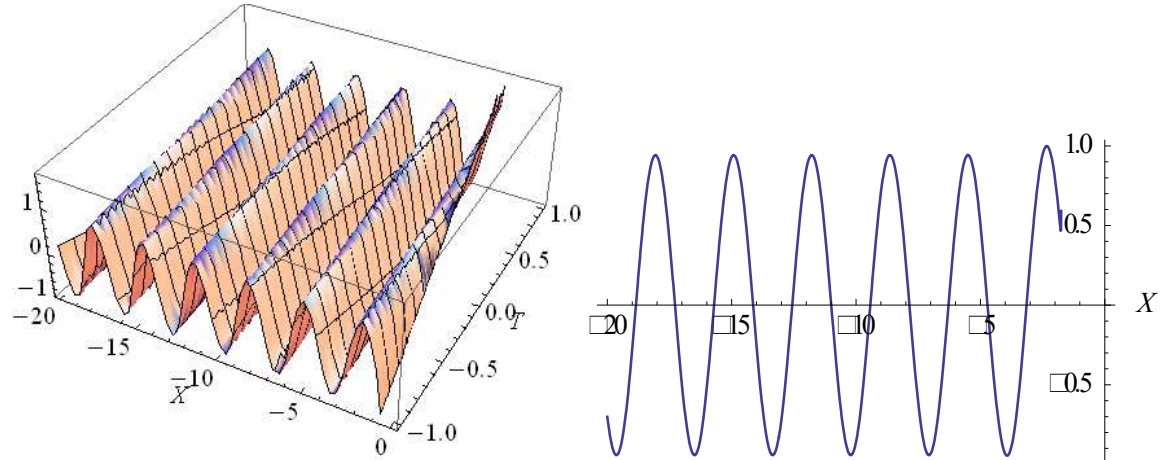

Figure 16. The cubic-soliton of the imaginary part Eq.(96) in 2D and 3D with values: $\zeta_{0}=1$

$$
A_{-2}=0, A_{-1}=0.1, A_{0}=-0.7, a_{1}=2, a_{2}=4.5, A_{1}=2.1, A_{2}=1.3, m=10.4
$$

By the same manners we can plot the other solutions after simplifying it.

Moreover, we can implement the first and the second family of the proposed method for the imaginary part of the power-low nonlinearity Eq.(67) to extract the cubic- solitons.

\section{Description of the SWAM}

To construct the solution in the framework of the SWAM [32, 34], let us admit this wave transformation,

$$
Q(x, t)=\psi(x, t) e^{i R(x, t)}
$$

Where $\psi(x, t)$ is the portion amplitude, while $R(x, t)$ is the phase portion of soliton.

Via simple calculations we can extract the following relations,

$$
\begin{aligned}
& Q_{t}=\left(\psi_{t}+i \psi R_{t}\right) e^{i R} \\
& Q_{x}=\left(\psi_{x}+i \psi R_{x}\right) e^{i R} \\
& Q_{x x}=\left(\psi_{x x}+2 i \psi_{x} R_{x}+i \psi R_{x x}-\psi R_{x}^{2}\right) e^{i R} \\
& Q_{x x x}=\left(\psi_{x x x}+3 i R_{x} \psi_{x x}+3 i R_{x x} \psi_{x}+i R_{x x x} \psi-i R_{x}^{3} \psi-3 R_{x} R_{x x} \psi\right) e^{i R}
\end{aligned}
$$

The bright and dark cubic-soliton solutions can be constructed as follows,

(I) The bright cubic-soliton solutions

$$
\begin{aligned}
& \psi(x, t)=A_{1} \operatorname{sech}^{R_{1}}\left(t_{1}\right), \text { where } t_{1}=B\left(x-w_{1} t\right) \text { and } R_{1}(x, t)=k x-\Omega t \\
& \psi_{t}=-A_{1} B w_{1} R_{1} \operatorname{sech}^{R_{1}}\left(t_{1}\right) \tanh \left(t_{1}\right)
\end{aligned}
$$




$$
\begin{gathered}
\psi_{x}=A_{1} B R_{1} \operatorname{sech}^{R_{1}}\left(t_{1}\right) \tanh \left(t_{1}\right) \\
\psi_{x x}=A_{1} B^{2} R_{1}\left(1+R_{1}\right) \operatorname{sech}^{R_{1}+2}\left(t_{1}\right)-A_{1} B^{2} R_{1}^{2} \operatorname{sech}^{R_{1}}\left(t_{1}\right) \\
\psi_{x x x}=A_{1} B^{3} R_{1}\left(R_{1}+1\right)\left(R_{1}+2\right) \operatorname{sech}^{R_{1}+2}\left(t_{1}\right) \tanh \left(t_{1}\right)-A_{1} B^{3} R_{1}^{3} \operatorname{sech}^{R_{1}}\left(t_{1}\right) \tanh \left(t_{1}\right)
\end{gathered}
$$

(II) The dark soliton solutions

$$
\begin{gathered}
\psi(x, t)=A_{2} \tanh ^{R_{2}}\left(t_{2}\right), \text { where }_{2}=B\left(x-w_{2} t\right) \text { and } R_{2}(x, t)=k x-\Omega t \\
\psi_{t}=-A_{2} B R_{2} w_{2}\left[\tanh ^{R_{2}-1}\left(t_{2}\right)-\tanh ^{R_{2}+1}\left(t_{2}\right)\right] \\
\psi_{x}=A_{2} B R_{2}\left[\tanh ^{R_{2}-1}\left(t_{2}\right)-\tanh ^{R_{2}+1}\left(t_{2}\right)\right] \\
\psi_{x x}=A_{2} R_{2}\left(R_{2}-1\right) B^{2} \tanh ^{R_{2}-2}\left(t_{2}\right)-2 A_{2} R_{2}^{2} B^{2} \tanh ^{R_{2}}\left(t_{2}\right)+ \\
A_{2} R_{2}\left(R_{2}+1\right) B^{2} \tanh ^{R_{2}+2}\left(t_{2}\right) \\
\psi_{x x x}=A_{2} B^{3} R_{2}\left(R_{2}-1\right)\left(R_{2}-2\right) \tanh ^{R_{2}-3}\left(t_{2}\right)-\left[A_{2} B^{3} R_{2}\left(R_{2}-1\right)\left(R_{2}-2\right)+2 A_{2} R_{2}^{3} B^{3}\right] \tanh ^{R_{2}-1}\left(t_{2}\right)+ \\
{\left[A_{2} B^{3} R_{2}\left(R_{2}+1\right)\left(R_{2}+2\right)+2 A_{2} R_{2}^{3} B^{3}\right] \tanh ^{R_{2}+1}\left(t_{2}\right)-A_{2} B^{3} R_{2}\left(R_{2}+1\right)\left(R_{2}+2\right) \tanh ^{R_{2}+3}\left(t_{2}\right)}
\end{gathered}
$$

\section{The bright and dark cubic-solitons for kerr-law nonlinearity}

\subsection{The bright cubic-solitons for kerr-law nonlinearity}

Via substituting the relations (97-101) into Eq. (12) mentioned above we obtain,

$$
\begin{array}{r}
i\left(\psi_{t}-i \Omega \psi\right) e^{i R_{1}}+\psi^{3} e^{i R_{1}}+i a\left(\psi_{x x x}+3 i k \psi_{x x}-i k^{3} \psi\right) e^{i R_{1}}= \\
i\left[v \psi^{2}\left(\psi_{x}+i k \psi\right)+\lambda\left(3 k \psi^{2} \psi_{x}+i k \psi^{3}\right)+2 \mu k \psi_{x} \psi^{2}\right] e^{i R_{1}}
\end{array}
$$

This equation will be divided into the following real and imaginary parts respectively:

$$
\begin{aligned}
& 3 a k \psi_{x x}-(1+k v+k \lambda) \psi^{3}-\left(\Omega+a k^{3}\right) \psi=0, \\
& a \psi_{x x x}-(3 k \lambda+2 \mu k+v) \psi^{2} \psi_{x}+\psi_{t}=0 .
\end{aligned}
$$

Now, via inserting the relations (102-106) in the real and imaginary parts equations (113) and (114), we get:

$$
\begin{aligned}
& \quad 3 a k A_{1} B^{2} R_{1}\left(1+R_{1}\right) \operatorname{sech}^{R_{1}+2}\left(t_{1}\right)-A_{1}\left(B^{2} R_{1}^{2}+\Omega+a k^{3}\right) \operatorname{sech}^{R_{1}}\left(t_{1}\right) \\
& \quad-(1+k v+k \lambda) A_{1}^{3} \operatorname{sech}^{3 R_{1}}\left(t_{1}\right)=0, \\
& a\left(A_{1} B^{3} R_{1}\left(R_{1}+1\right)\left(R_{1}+2\right) \operatorname{sech}^{R_{1}+2}\left(t_{1}\right) \tanh \left(t_{1}\right)-A_{1} B^{3} R_{1}^{3} \operatorname{sech}^{R_{1}}\left(t_{1}\right) \tanh \left(t_{1}\right)\right)- \\
& (3 k \lambda+2 \mu k+v) A_{1}^{3} B R_{1} \operatorname{sech}^{R_{1}+2}\left(t_{1}\right) \tanh \left(t_{1}\right)-A_{1} B w_{1} R_{1} \operatorname{sech}^{R_{1}}\left(t_{1}\right) \tanh \left(t_{1}\right)=0 .
\end{aligned}
$$

Via equating the highest order powers $\operatorname{sech}^{i}\left(t_{1}\right)$ of the real part we get $R_{1}=1$ and hence,

$$
A_{1}= \pm \sqrt{\frac{6 a k B^{2}}{1+k v+\lambda k}} \text {, by substituting at the imaginary part we get } \Omega=-2, B= \pm i \sqrt{w_{1}} \text {. }
$$

Now, according to these obtained constants the solution in the framework of the proposed method is,

$$
Q= \pm \sqrt{\frac{6 a k B^{2}}{1+k v+\lambda k}} \operatorname{sech} B\left(x-w_{1} t\right) e^{i(k x-\Omega t)}
$$




$$
Q= \pm 2 i \operatorname{sech} \pm i(x-t) e^{i(x+2 t)}
$$

This admits these four solutions which are,

$$
\begin{aligned}
& \text { (1) } Q=2 i(\operatorname{sech}[i(x-t)]) e^{i(x+2 t)} \\
& \text { (2) } Q=-2 i(\operatorname{sech}[-i(x-t)]) e^{i(x+2 t)} \\
& \text { (3) } Q=2 i(\operatorname{sech}[-i(x-t)]) e^{i(x+2 t)} \\
& \text { (4) } Q=-2 i(\operatorname{sech}[i(x-t)]) e^{i(x+2 t)}
\end{aligned}
$$

Now, let us plot only two of them, say the first and the fourth

(1) in which is $Q=2 i(\operatorname{sech}[i(x-t)]) e^{i(x+2 t)}$

$$
\begin{aligned}
& \operatorname{Re} Q=\frac{-2 \cos (x+2 t)}{\cos (x-t)} \\
& \operatorname{Im} Q=\frac{-2 \sin (x+2 t)}{\cos (x-t)}
\end{aligned}
$$
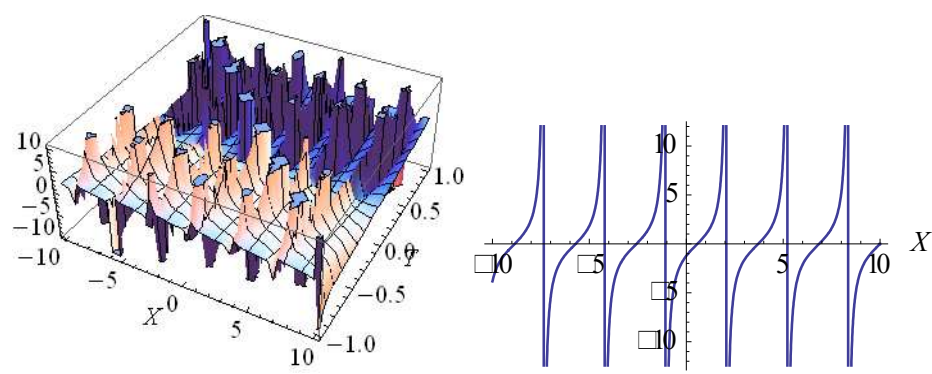

Figure 17. The bright cubic-soliton of the real part Eq.(120) in 2D and 3D with values:

$$
A_{1}=2 i, B=i \sqrt{w_{1}}, R_{1}=1, \Omega=-2, a=k=w_{1}=v=\lambda=\mu=1, t=.5 \text { for } 2 D
$$

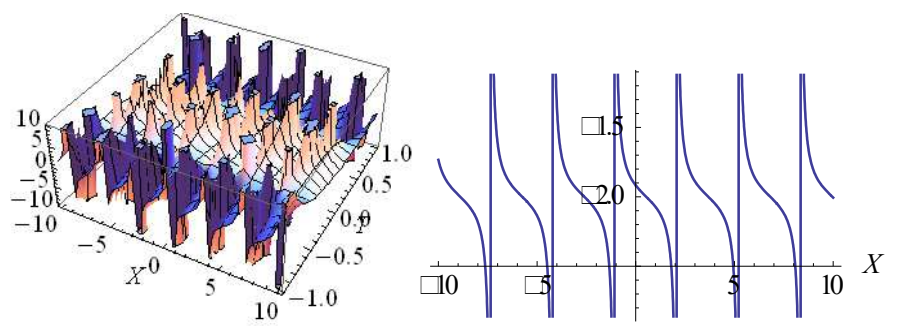

Figure 18. The bright cubic-soliton of the imaginary part Eq.(121) in $2 \mathrm{D}$ and $3 \mathrm{D}$ with values:

$$
A_{1}=2 i, B=i \sqrt{w_{1}}, R_{1}=1, \Omega=-2, a=k=w_{1}=v=\lambda=\mu=1, t=.5 \text { for } 2 D
$$

(2) in which is $Q=-2 i(\operatorname{sech}[i(x-t)]) e^{i(x+2 t)}$

$$
\begin{aligned}
& \operatorname{Re} Q=\frac{2 \cos (x+2 t)}{\cos (x-t)} \\
& \operatorname{Im} Q=\frac{2 \sin (x+2 t)}{\cos (x-t)}
\end{aligned}
$$



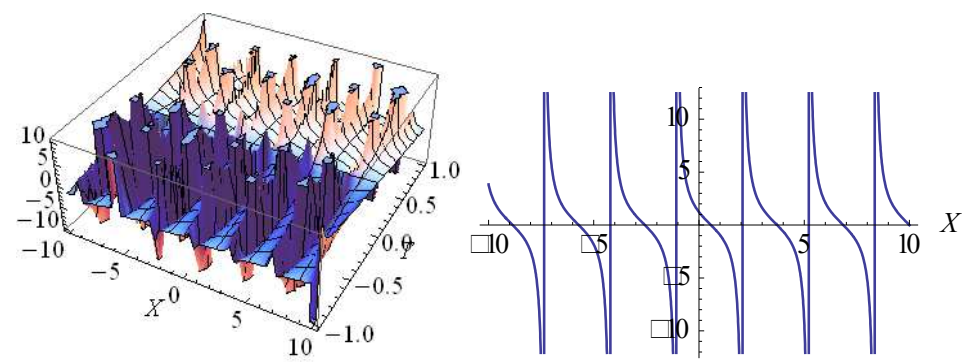

Figure 19. The bright cubic-soliton of the real part Eq.(122) in 3D and $2 \mathrm{D}$ with values: $A_{1}=-2 i, B=i \sqrt{w_{1}}, R_{1}=1, \Omega=-2, a=k=w_{1}=v=\lambda=\mu=1, t=.5$ for $2 D$
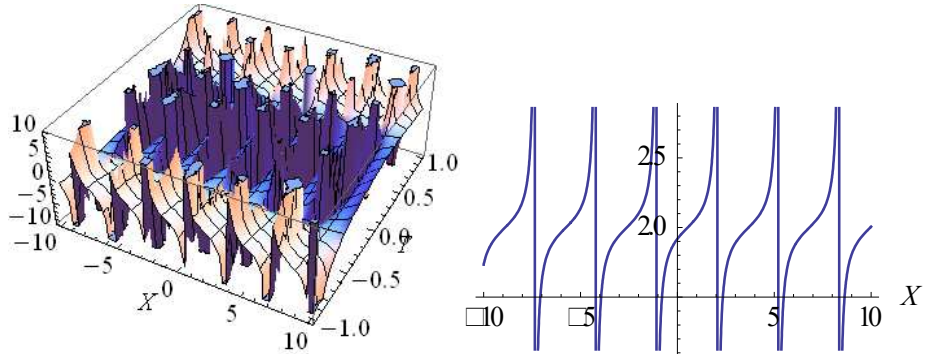

Figure 20. The bright cubic-soliton of the imaginary part Eq.(123) in $2 \mathrm{D}$ and $3 \mathrm{D}$ with values:

$$
A_{1}=-2 i, B=i \sqrt{w_{1}}, R_{1}=1, \Omega=-2, a=k=w_{1}=v=\lambda=\mu=1, t=.5 \text { for } 2 D
$$

By the same method we can plot the other two solutions

5.2 The dark cubic solitons for kerr-low nonlinearity Now, via inserting the relations (107-111) into the real and imaginary parts equations (113), (114) we obtain:

$$
\begin{aligned}
& 3 a k A_{2} R_{2}\left(R_{2}-1\right) B^{2} \tanh ^{R_{2}-2}\left(t_{2}\right)-\left[6 a k A_{2} R_{2}^{2} B^{2}+\left(\Omega+a k^{3}\right) A_{2}\right] \tanh ^{R_{2}}\left(t_{2}\right) \\
& +3 a k A_{2} R_{2}\left(R_{2}+1\right) B^{2} \tanh ^{R_{2}+2}\left(t_{2}\right)-(1+k v+k \lambda) A_{2}^{3} \tanh ^{3 R_{2}}\left(t_{2}\right)=0, \\
& \left.a A_{2} B^{3} R_{2}\left(R_{2}-1\right)\left(R_{2}-2\right) \tanh ^{R_{2}-3}\left(t_{2}\right)-a A_{2} B^{3} R_{2}\left(R_{2}+1\right)\left(R_{2}+2\right)\right] \tanh ^{R_{2}+3}\left(t_{2}\right) \\
& -\left\{A_{2} B R_{2} w_{2}+a A_{2} B^{3} R_{2}\left(R_{2}-1\right)\left(R_{2}-2\right)+2 a A_{2} R_{2}^{3} B^{3}\right\} \tanh ^{R_{2}-1}\left(t_{2}\right) \\
& +\left\{A_{2} B R_{2} w_{2}+a A_{2} B^{3} R_{2}\left(R_{2}+1\right)\left(R_{2}+2\right)+2 a A_{2} R_{2}^{3} B^{3}\right\} \tanh ^{R_{2}+1}\left(t_{2}\right) \\
& +(3 k \lambda+2 \mu k+v) A_{2}^{3} B R_{2} \tanh ^{3 R_{2}+1}\left(t_{2}\right)-(3 k \lambda+2 \mu k+v) A_{2}^{3} B R_{2} \tanh ^{3 R_{2}-1}\left(t_{2}\right)=0 .
\end{aligned}
$$

Via equating the highest order powers $\tanh ^{i}\left(t_{1}\right)$ of the real part we get $R_{2}=1$ and hence, $A_{2}= \pm \sqrt{\frac{-2 w_{2}}{1+k v+\lambda k}}$, by substituting at the imaginary part we get $\Omega=-0.75, B= \pm 0.5 i, \mu=-0.5$.

Now, according to these obtained constants the solution in the framework of the proposed method is,

$$
\begin{aligned}
& Q= \pm \sqrt{\frac{-2 w_{2}}{1+k v+\lambda k}} \tanh B\left(x-w_{2} t\right) e^{i(k x-\Omega t)} \\
& Q= \pm \sqrt{\frac{1}{3}} \tanh \pm i(x+0.5 t) e^{i(x+0.75 t)}
\end{aligned}
$$




$$
\begin{aligned}
& Q= \pm \sqrt{\frac{1}{3}} i \tan (x+0.5 t) e^{i(x+0.75 t)} \\
& Q= \pm 0.6 i \tan (x+0.5 t)[\cos (x+0.75)+i \sin (x+0.75)] \\
& \operatorname{Re} Q= \pm 0.6 \tan (x+0.5 t) \times \sin (x+0.75) \\
& \operatorname{Im} Q= \pm 0.6 \tan (x+0.5 t) \times \cos (x+0.75)
\end{aligned}
$$

For similarity we will plot only one solution to each part
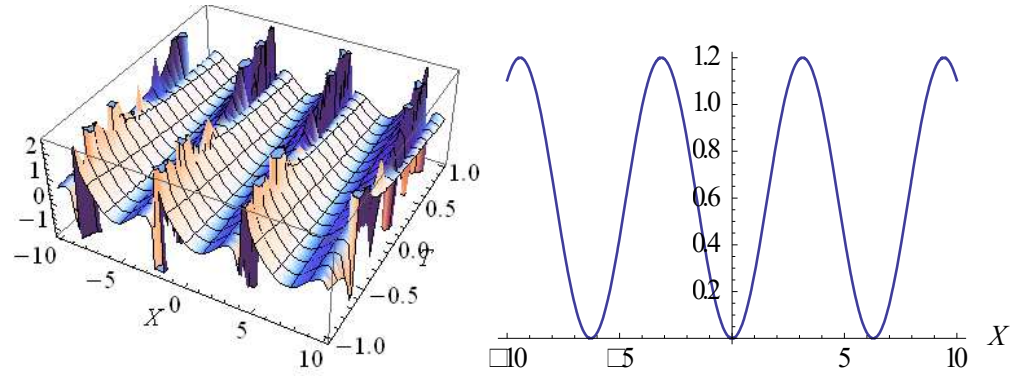

Figure 21. The dark cubic-soliton of the real part Eq.(128) in 3D and 2D with values: $A_{1}=0.6 i, B=0.5 i, R_{2}=1, \Omega=-0.75, a=k=w_{1}=v=\lambda=1, \mu=-0.5$,

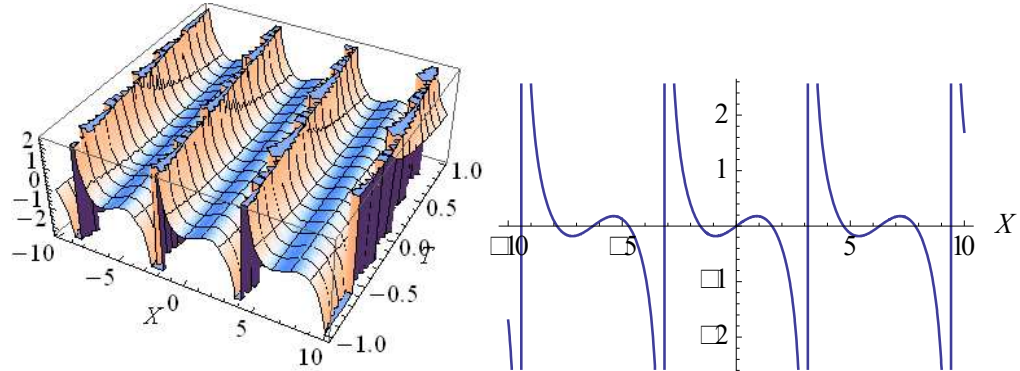

Figure 22. The dark cubic-soliton of the imaginary part Eq.(129) in 2D and 3D with values: $A_{1}=0.6 i, B=0.5 i, R_{2}=1, \Omega=-0.75, a=k=w_{1}=v=\lambda=1, \mu=-0.5$,

By the same manner we can plot the other solutions.

\section{The bright and dark cubic-solitons for power-law nonlinearity}

6.1 The bright cubic-solitons for power-law nonlinearity Via inserting the relations (97-101) into Eq. (65) mentioned above we obtain,

$$
\begin{aligned}
& \operatorname{Re}-3 a k \psi_{x x}+\left(\Omega+a k^{3}\right) \psi+(1+k v+k \lambda) \psi^{2 m+1}=0 . \\
& \operatorname{Im} a \psi_{x x x}-[v+(2 m+1) \lambda+2 m \mu] \psi^{2 m} \psi_{x}+\psi_{t}=0 . \\
& -3 a k A_{1} B^{2} R_{1}\left(1+R_{1}\right) \operatorname{sech}^{R_{1}+2}\left(t_{1}\right)+\left(\left(\Omega+a k^{3}\right) A_{1}+3 a k A_{1} B^{2} R_{1}^{2}\right) \operatorname{sech}^{R_{1}}\left(t_{1}\right)+ \\
& \quad(1+k v+k \lambda) A_{1}^{2 m+1} \operatorname{sech}^{(2 m+1) R_{1}}\left(t_{1}\right)=0 . \\
& a A_{1} B^{3} R_{1}\left(R_{1}+1\right)\left(R_{1}+2\right) \operatorname{sech}^{R_{1}+2}\left(t_{1}\right) \tanh \left(t_{1}\right)-\left(A_{1} B w_{1} R_{1}+a A_{1} B^{3} R_{1}^{3}\right) \operatorname{sech}^{R_{1}}\left(t_{1}\right) \tanh \left(t_{1}\right) \\
& -[v+(2 m+1) \lambda+2 m \mu] A_{1}^{2 m+1} B R_{1} \operatorname{sech}^{(2 m+1) R_{1}}\left(t_{1}\right) \tanh \left(t_{1}\right)=0 .
\end{aligned}
$$


Via equating the highest order powers $\operatorname{sech}^{i}\left(t_{1}\right)$ of the real part we get $R_{1}=\frac{1}{m}$ hence, $A_{1}= \pm \frac{1}{m} \sqrt{\frac{a(1+m)(2 m+1)}{(v+(2 m+1) \lambda+2 m \mu)}}, \quad \Omega=-\frac{3 w_{1} k+a m^{2} k^{3}}{m^{2}}$ and by substituting at the imaginary part we get $B= \pm i \sqrt{\frac{w_{1}}{a}}$

Now, according to the previous values of constants for the kerr-law which are $B= \pm i$, $a=k=w_{1}=v=\lambda=\mu=1$ will lead to $\Omega=-\frac{3+m^{2}}{m^{2}}, A_{1}= \pm \frac{1}{m} \sqrt{\frac{(1+m)}{2}}$ for the power-low constants.

Hence,

$$
\Omega=-\frac{3+m^{2}}{m^{2}}, B= \pm i, A_{1}=A_{1}= \pm \frac{1}{m} \sqrt{\frac{(1+m)}{2}}, R_{1}=\frac{1}{m}, a=k=w_{1}=v=\lambda=\mu=1 .
$$

Consequently, the solution in the framework of the proposed method is,

$$
\begin{aligned}
& Q= \pm \frac{1}{m} \sqrt{\frac{(1+m)}{2}} \operatorname{sech} \pm i(x-t) e^{i\left(x+\frac{3+m^{2}}{m^{2}} t\right)} \\
& \operatorname{Re} Q= \pm \frac{\sin (x+1.3 t)}{2 \cos (x-t)}, m=3 .
\end{aligned}
$$
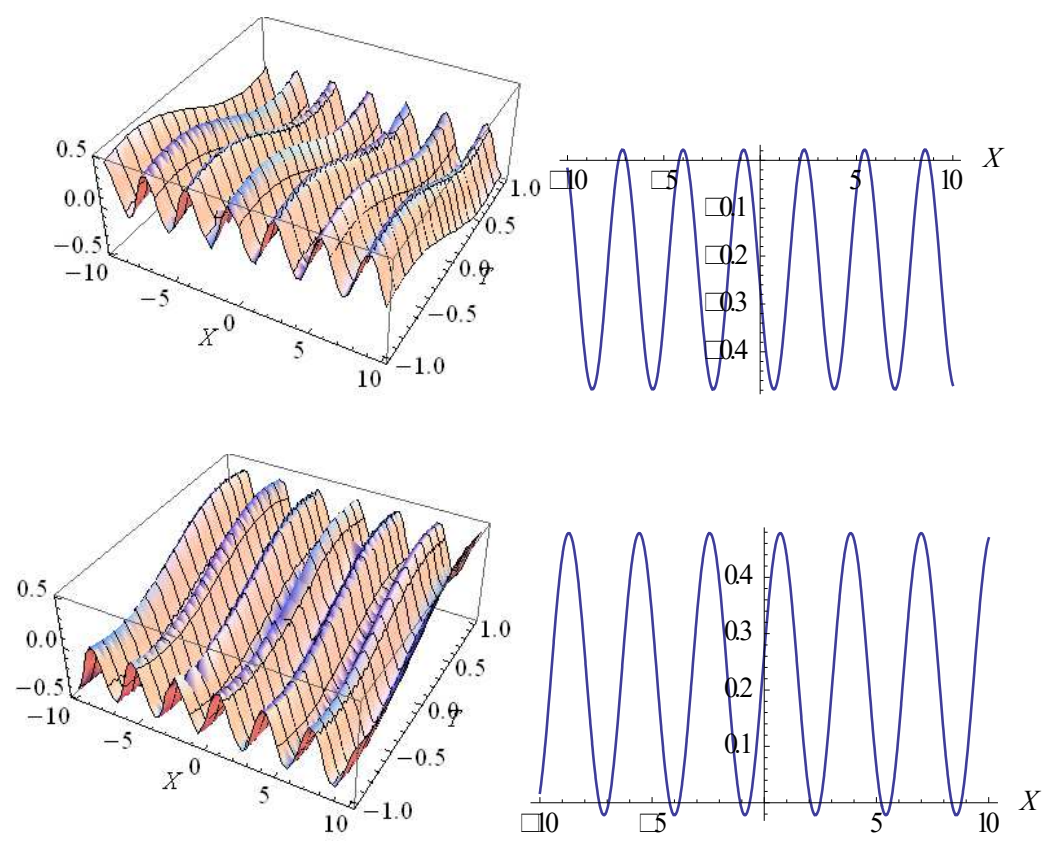

Figure 23. The bright cubic-soliton of the real part Eq.(135) in 3D and 2D with values: $\Omega=-1.3, B= \pm i, A_{1}= \pm 0.5, R_{1}=0.3, m=3, a=k=w_{1}=v=\lambda=\mu=1, t=0.5$ for $2 D$

$$
\operatorname{Im} Q= \pm \frac{\cos (x+1.3 t)}{2 \cos (x-t)}, m=3
$$



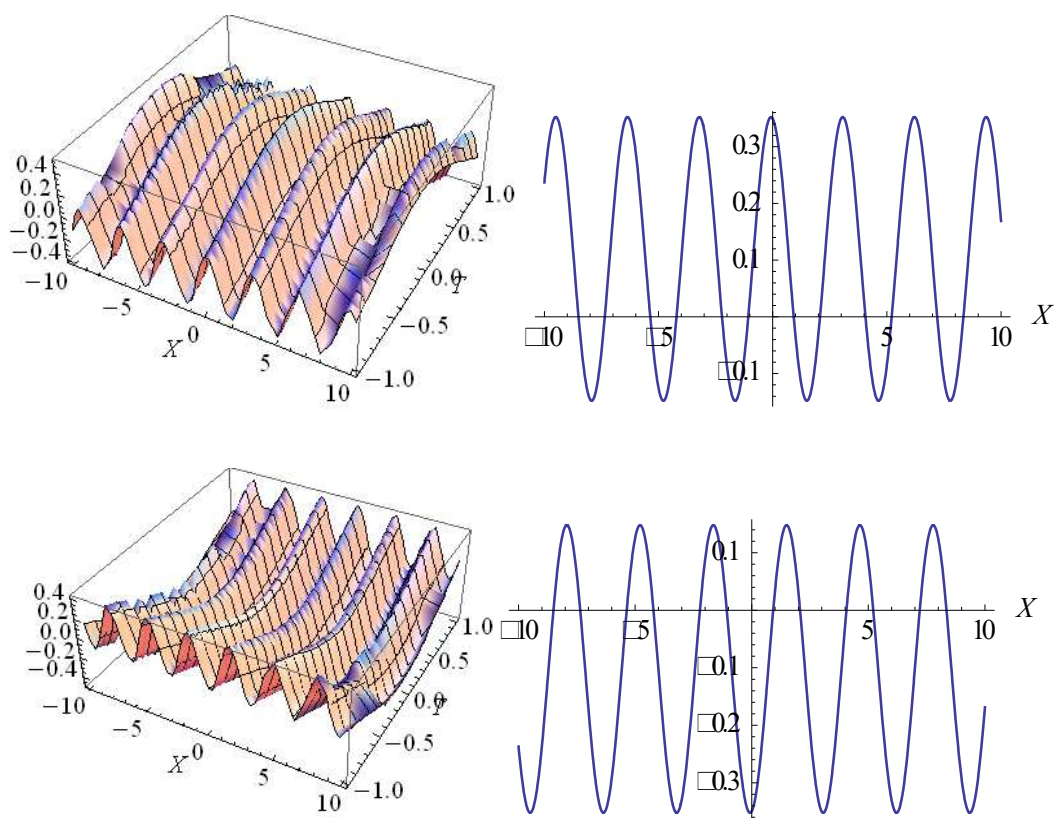

Figure 24. The bright cubic-soliton of the imaginary part Eq.(137) in 3D and 2D with values: $\Omega=-1.3, B= \pm i, A_{1}= \pm 0.5, R_{1}=0.3, m=3, a=k=w_{1}=v=\lambda=\mu=1, t=.5$ for $2 \mathrm{D}$

6.2 The dark cubic solitons for power law-nonlinearity Via inserting the relations (107-111) into Eq. (130) and Eq. (131) mentioned above we get,

$$
\begin{aligned}
& -3 a k A_{2} R_{2}\left(R_{2}-1\right) B^{2} \tanh ^{R_{2}-2}\left(t_{2}\right)+\left(\left(\Omega+a k^{3}\right) A_{2}+6 a k A_{2} R_{2}^{2} B^{2}\right) \tanh ^{R_{2}}\left(t_{2}\right) \\
& -3 a k A_{2} R_{2}\left(R_{2}+1\right) B^{2} \tanh ^{R_{2}+2}\left(t_{2}\right)+(1+k v+k \lambda) A_{2}^{2 m+1} \tanh ^{(2 m+1) R_{2}}\left(t_{2}\right)=0 . \\
& a A_{2} B^{3} R_{2}\left(R_{2}-1\right)\left(R_{2}-2\right) \tanh ^{R_{2}-3}\left(t_{2}\right)-a A_{2} B^{3} R_{2}\left(R_{2}+1\right)\left(R_{2}+2\right) \tanh ^{R_{2}+3}\left(t_{2}\right)- \\
& {\left[a A_{2} B^{3} R_{2}\left(R_{2}-1\right)\left(R_{2}-2\right)+2 a A_{2} R_{2}^{3} B^{3}-A_{2} B R_{2}\right] \tanh ^{R_{2}-1}\left(t_{2}\right)+} \\
& {\left[a A_{2} B^{3} R_{2}\left(R_{2}+1\right)\left(R_{2}+2\right)+a 2 A_{2} R_{2}^{3} B^{3}+A_{2} B R_{2}\right] \tanh ^{R_{2}+1}\left(t_{2}\right)} \\
& -A_{2}^{2 m+1} B R_{2}[v+(2 m+1) \lambda+2 m \mu]\left(\tanh ^{(2 m+1) R_{2}-1}\left(t_{2}\right)-\tanh ^{(2 m+1) R_{2}+1}\left(t_{2}\right)\right)=0 .
\end{aligned}
$$

Via equating the highest order powers $\tanh ^{i}\left(t_{2}\right)$ of the real part we get $R_{2}=\frac{1}{m}$ and hence,

$\Omega+a k^{3}=\frac{-6 a k B^{2}}{m^{2}}$, by substituting at the imaginary part we get, $A_{2}^{2 m}=\sqrt{\frac{3 a k B^{2}(1+m)}{m^{2}(1+k v+k \lambda)}}$

Now, according to the same values of constants of the bright solutions $a=k=w_{1}=v=\lambda=\mu=1, m=3$, the above constants of the dark solutions become $\Omega=-7$, $A_{1}= \pm 0.2 \sqrt{\frac{4}{9}}, B=1$, hence the dark solutions in the framework of the proposed method are,

$$
\begin{aligned}
& Q= \pm 0.02 \tanh (x-t) e^{i(x-7 t)} \\
& \operatorname{Re} Q= \pm 0.02 \tanh (x-t) \times \cos (x-7 t) \\
& \operatorname{Im} Q= \pm 0.02 \tanh (x-t) \times \sin (x-7 t)
\end{aligned}
$$



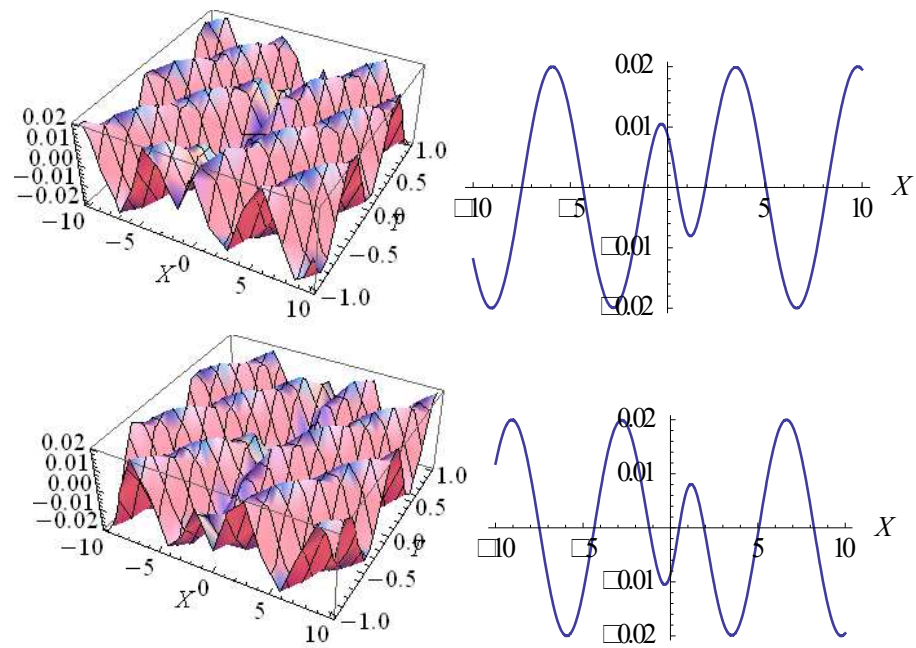

Figure 25. The dark cubic-solitons of the real part Eq.(141) in $3 \mathrm{D}$ and $2 \mathrm{D}$ with values:

$$
\Omega=-7, B=1, A_{1}= \pm 0.02, R_{1}=0.3, m=3, a=k=w_{1}=v=\lambda=\mu=1, t=.5 \text { for } 2 \mathrm{D}
$$
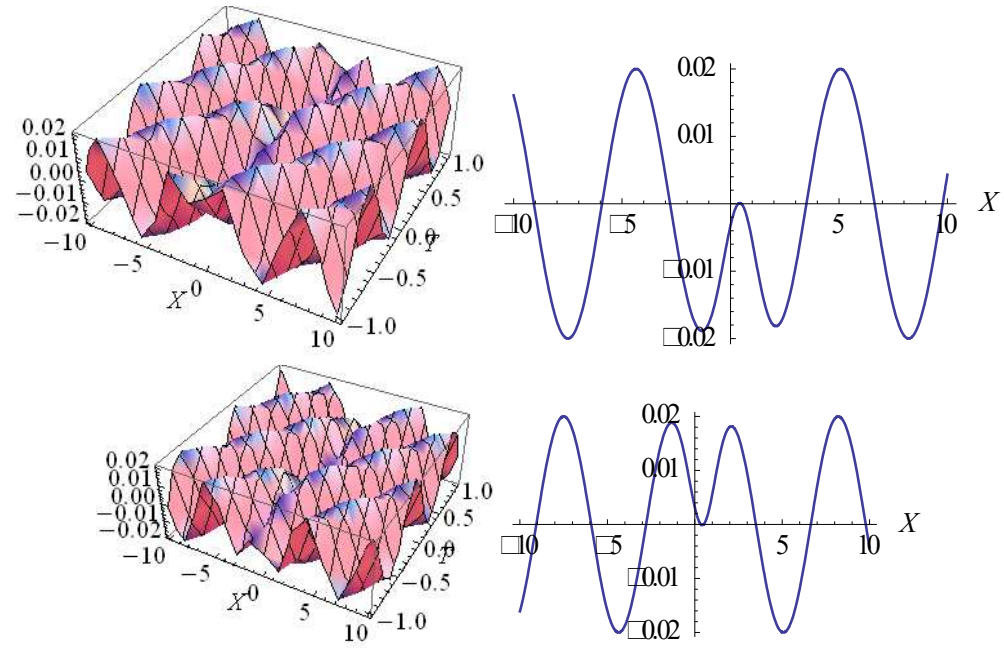

Figure 26. The dark cubic-solitons of the imaginary part Eq.(142) in $3 \mathrm{D}$ and $2 \mathrm{D}$ with values:

$$
\Omega=-7, B=1, A_{1}= \pm 0.02, R_{1}=0.3, m=3, a=k=w_{1}=v=\lambda=\mu=1, t=.5 \text { for } 2 \mathrm{D}
$$

\section{9-Conclusion}

From the power point of view for two important and powerful distinct techniques new accurate cubic- solitons for the Kerr-low and Power-low NLSE in the presence of third-order dispersion effects, absence of the chromatic dispersion have been extracted. The two techniques have been implemented in the same vein and parallel. The first one is the ESEM which has a successful history in extracting the optical soliton solutions for many nonlinear phenomenas arising in different branches of science. This schema is applied perfectly to introduce new impressive and accurate visions of the cubic solitons for the Kerr-law and Power-law NLSE that involve the third-order dispersion effect and exclude the chromatic dispersion effect, which are obviously through Figures (1-16). In related subject the SWAM has been applied effectively to establish other new accurate perceptions of the cubic solitons for the kerr-law and power law nonlinearity mentioned before, which are obviously through Figures (17-26). Our new achieved pictures of the accurate cubic soliton solutions in the framework of these two various manners which weren't achieved before by any other authors denote to the novelty of these results, especially compared with that achieved previously by [6-8] and [33-34] who applied different techniques to study these two cases significantly. Consequently, new distinct and impressive visions to the cubic solitons of these two different versions of this model have been demonstrated. Moreover, the achieved cubic solitons via 
these two different algorithms will add good future studies not only for this model but also for all related phenomenas.

\section{References:}

[1] Bekir, A., Zahran, E.H.M., Bright and dark soliton solutions for the complex KunduEckhaus equation; Optik-International Journal for Light and Electron Optics 223; (2020) 165233

[2] Biswas, A.; 1-Soliton solution of the K(m; n) equation with generalized evolution, Phys. Lett. A; 372; (2008) 4601-4602

[3] Triki, H., Wazwaz, A.M.; Bright and dark soliton solutions for a $\mathrm{K}(\mathrm{m}$; $\mathrm{n})$ equation with tdependent coefficients, Phys. Lett. A; 373; (2009) 2162-2165

[4] Triki, H., Wazwaz, A.M.; Bright and dark solitons for a generalized Korteweg-de Vriesmodified Korteweg-de Vries equation with high-order nonlinear terms and time-dependent coefficients, Can. J. Phys. 89; (2011) 253-259

[5] Liu, C.; Exact solutions for the higher-order nonlinear Schrodinger equation in nonlinear optical fibres. Chaos, Solitons and Fractals 23; (2005) 949-955

[6] Xiao, L.L., Liang, W.M.; The (G'/G)-Expansion Method and travelling wave solutions for a higher-order nonlinear Schrodinger equation, Appl. Math. Comput.208; (2009) 440-445

[7] El-Ganaini, S.I.A.; The first integral method to the nonlinear Schrodinger equations in higher dimensions; Abst. Appl. Anal. 10; (2013) 349173

[8] Mirzazadeh, M., Eslami, M., and Biswas, A.; Dispersive optical solitons by Kudryashov's method; Optik, 125; 23; (2014) 6874-6880

[9] Blanco-Redondo, A., Sterke, C.M.d., Sipe, J.E., Krauss, T.F., Eggleton, B.J., and Husko, C.; Pure-quartic solitons, Nat. Commun. 7; (2016) 10427

[10] Bekir,A., Zahran, E.H.M.; Three distinct and impressive visions for the soliton solutions to the higher-order nonlinear Schrodinger equation; Optik - International Journal for Light and Electron Optics 228; (2021) 166157

[11] Das, A., Biswas, A., Ekici, M., Khan, S., Zhou, Q., Moshokoa, S.P., Suppressing internet bottleneck with fractional temporal evolution of cubic-quartic optical solitons, Optik 182; (2019) 303-307

[12] Bansal, A., Biswas, A., Zhou, Q., Babatin, M.M., Lie symmetry analysis for cubicquartic nonlinear Schr"odinger's equation, Optik 169; (2018) 12-15.

[13] Biswas, A., Triki, H., Zhou, Q., Moshokoa, S.P., Ullah, M.Z., Belic, M., Cubic-quartic optical solitons in Kerr and power law media, Optik 144; (2017) 357-362.

[14] Biswas, A., Kara, A.H., Ullah, M.Z., Zhou, Q., Triki, H., Belic, M., Conservation laws for cubic-quartic optical solitons in Kerr and power law media, Optik 145; (2017) 650-654.

[15] Biswas, A., Arshed, S., Application of semi-inverse variational principle to cubic-quartic optical solitons with Kerr and power law nonlinearity, Optik 172; (2018) 847-850.

[16] Gonzalez-Gaxiola, O., Biswas, A., Mallawi, F., Belic, M.; Cubic-quartic bright optical solitons by improved Adomian decomposition method, J. Adv. Res. 21; (2020) 161-167.

[17] Kohl, R.W., Biswas, A.,Ekici, M., Zhou, Q., Moshokoa, S.P., Belic, M.R.; Cubic-quartic optical soliton perturbation by semi-inverse variational principle, Optik 185; (2019) 45-49.

[18] Yildirim, Y., Biswas, A., Guggilla, P., Mallawi, F., Belic, M.R., Cubic-quartic optical solitons in birefringent fibers with four forms of nonlinear refractive index, Optik 203; (2020) 163885

[19] Yildirim, Y., Biswas, A.,Jawad, A.J.M., Ekici, M., Zhou, Q., Khan, S., Alzahrani, A.K., and Belic, M.R., Cubic-quartic optical solitons in birefringent fibers with four forms of nonlinear refractive index by exp-function expansion, Results Phys.16; (2020) 102913

[20] Wazwaz, A.M., and Xu, G.Q.; Bright, dark, and Gaussons optical solutions for fourth order Schrodinger equation with cubic-quantic and logarithmic nonlinearities, Optik; 202; (2020) 163564 
[21] Huang, D.J., Li, D.S., Zhang, H.Q., Explicit and exact travelling wave solutions for the generalized derivative Schr"odinger equation, Chaos Solitons Fract. 31;(2007) 586-593

[22] Kudryashov, N.A., Mathematical model of propagation pulse in optical fiber with power nonlinearities, Optik, 212; (2020) 164750

[23] Kudryashov, N.A., Solitary wave solutions of hierarchy with non-local nonlinearity, Appl. Math. Lett. 103; (2020) 106155

[24] Kudryashov, N.A., Highly dispersive solitary wave solutions of perturbed nonlinear Schr"odinger equations, Appl. Math. Comput.; 371; (2020) 124972

[25] Kudryashov, N.A., Highly dispersive optical solitons of the generalized nonlinear eighthorder Schrödinger equation, Optik 206; (2020) 164335.

[26] Li, B., Zhao, J., Pan, A., Mirzazadeh, M., Ekici, M., Zhou, Q., Liu, W., Stable propagation of optical solitons in fiber lasers by using symbolic computation, Optik 178; (2019) 142-145.

[27] Bekir,A., Zahran, E.H.M. ; New visions of the soliton solutions to the modified nonlinear Schrodinger equation; Optik; 232 (2021) 166539

[28] Bekir ,A., Zahran, E.H.M., New vision for the soliton solutions to the complex Hirotadynamical model; Physica Scripta; 96 (2021) 055212

[29] Bekir, A.,Shehata,M.S.M., and Zahran, E.H.M; Comparison between the new exact and numerical solutions of the Mikhailov-Novikov-Wang equation, accepted for publication in the Numerical Method of Partial Differential Equation journal; (in press).

[30] Shehata, M.S.M., Rezazadeh, H., Jawad, A.J.M., Zahran, E.H.M., Bekir,A.; Optical solitons to a perturbed Gerdjikov-Ivanov equation using two different techniques, accepted for publication in Revista Mexicana de Física; (in press).

[31] Bekir ,A., Zahran, E.H.M.; Painleve approach and its applications to get new exact solutions of three biological models instead of its numerical solutions; International Journal of Modern Physics B; 34, 29 (2020) 2050270

[32] Bekir,A., Zahran,E.H.M.;Optical soliton solutions of the thin-film ferro-electric materials equation according to the Painlevé approach, has been accepted for publication in Optical and Quantum Electronics; 53 (2021) 118.

[33] Hosseini, K., Osman, M.S., Mirzazadeh, M., and Rabiei, F., Investigation of different wave structures to the generalized third-order nonlinear Schrödinger equation, Optik 206; (2020); 164259.

[34] Yildırım, Y., Biswas, A., Asma, M., Guggilla, P., Khan, S., Ekici, M., Alzahrani, A.K., and Belic, M.R., Pure-cubic optical soliton perturbation with full nonlinearity ; Optik International Journal for Light and Electron Optics 222; (2020) 165394

[35] Kodama,Y., Hasegawa, A., Solitons in Optical Communications, Oxford University Press, Oxford, UK. (1995) 